\title{
Degenerate two-body and three-body coupled-channels systems: Renormalized effective Alt-Grassberger-Sandhas equations and near-threshold resonances
}

\author{
Atsunari Konishi \\ KEK Theory Center, IPNS, High Energy Accelerator Research Organization (KEK), 1-1 Oho, Tsukuba, Ibaraki 205-0801, Japan \\ Osamu Morimatsu \\ KEK Theory Center, IPNS, High Energy Accelerator Research Organization (KEK), 1-1 Oho, Tsukuba, Ibaraki 205-0801, Japan; \\ Department of Physics, Faculty of Science, University of Tokyo, 7-3-1 Hongo, Bunkyo-ku, Tokyo 113-0033, Japan; \\ and Department of Particle and Nuclear Studies, Graduate University for Advanced Studies (SOKENDAI), 1-1 Oho, Tsukuba, \\ Ibaraki 305-0801, Japan \\ Shigehiro Yasui \\ Department of Physics, Tokyo Institute of Technology, 2-12-1 Ohokayama, Meguro, Tokyo 152-8551, Japan
}

(Received 16 September 2017; revised manuscript received 24 November 2017; published 19 June 2018)

\begin{abstract}
Motivated by the existence of candidates for exotic hadrons whose masses are close to both two-body and three-body hadronic thresholds lying close to each other, we study degenerate two-body and three-body coupledchannels systems. We first formulate the scattering problem of non-degenerate two-body and three-body coupled channels as an effective three-body problem, i.e., as effective Alt-Grassberger-Sandhas (AGS) equations. We next investigate the behavior of $S$-matrix poles near the threshold when two-body and three-body thresholds are degenerate. We solve the eigenvalue equations of the kernel of AGS equations instead of AGS equations themselves to obtain the $S$-matrix pole energy. We then face a problem of unphysical singularity: although the physical transition amplitudes have physical singularities only, the kernels of AGS equations have unphysical singularities. We show, however, that these unphysical singularities can be removed by appropriate reorganization of the scattering equations and mass renormalization. The behavior of $S$-matrix poles near the degenerate threshold is found to be universal in the sense that the complex pole energy, $E$, is determined by a real parameter, $c$, as $c+E \log (-E)=0$, or equivalently, $c+\operatorname{Re} E \log (\operatorname{Re} E)=0$ and $\operatorname{Im} E=\pi \operatorname{Re} E / \log (\operatorname{Re} E)$. This behavior is different from that of either two-body or three-body systems and is characteristic of the degenerate two-body and three-body coupled-channels system. We expect that this new class of universal behavior might play a key role in understanding exotic hadrons.
\end{abstract}

DOI: 10.1103/PhysRevC.97.064001

\section{INTRODUCTION}

The $X(3872)$ was first observed in 2003 [1] and is considered not to be a simple charmonium [2-4] and is therefore a candidate for the exotic hadron. Since its mass is very close to the neutral $D \bar{D}^{*}$ threshold, it is pointed out that it has a significant $D \bar{D}^{*}$ molecular component [5-20]. However, we must be aware that its mass is also very close to the $D \bar{D} \pi$ three-body threshold. We therefore have to consider $D \bar{D}^{*}-D \bar{D} \pi$ hadronic two-body and three-body coupled-channels analysis whose importance has also been discussed [21-27]. See Refs. [28-31] for recent review articles of heavy quarkonium and candidates for the exotic hadron in those energy regions. There are both ex-

Published by the American Physical Society under the terms of the Creative Commons Attribution 4.0 International license. Further distribution of this work must maintain attribution to the author(s) and the published article's title, journal citation, and DOI. Funded by $S C O A P^{3}$. perimental and theoretical indications that a strange dibaryon exists in the $K^{-} p p$ system [32-34]. Since the resonance $\Lambda(1405)$ exists in the $\bar{K} N$ channel, $\bar{K} N N-\Lambda(1405) N-\pi \Sigma N$ two-body and three-body coupled-channels effects regarding $\Lambda(1405)$ as a compact $q q q$ baryon might play an important role in understanding the system. The existence of non-strange dibaryons was first discussed in the 1960s [35,36]; however, it is only recently that they were actually observed experimentally [37-43]. The importance of hadronic two-body and three-body coupled-channels analysis in these channels has also been discussed [44,45]. See, for example, an introduction in Ref. [46] for a review of the current status of non-strange dibaryon physics.

Two-body and three-body coupled-channels analysis is therefore required to deepen our understandings of those resonances whose thresholds lie close to each other. In this paper, motivated by such circumstances, we develop two-body and three-body coupled-channels scattering equations and investigate the $S$-matrix pole behavior near the thresholds in the case of a degenerate two-body and three-body coupledchannels system. 
The discussion so far has been focused on phenomenological aspects of hadron physics. However, the degenerate two-body and three-body coupled-channels system is also interesting from a purely theoretical perspective. It is known that the $S$-matrix pole behavior near the threshold in a singlechannel two-body and three-body system has a universal property [47]. Namely, it is determined by one or two parameters depending on how close the poles are located to the threshold. It is also known that universal behavior crucially depends on the phase-space property near the threshold, that is, whether it is a two-body system, a it is three-body system, or the system has relative angular momentum excitation [48]. Then, a question arises: How does the $S$-matrix pole behave near the thresholds in the case of a degenerate two-body and three-body coupled system? We expect a new class of universal behavior emerges in such a case. We might also want to ask the same question in relation to the Efimov effect. If two pairs of three particles develop a zero-energy bound state, an infinite number of bound states appear, which is known as the Efimov effect. A degenerate two-body and three-body coupled-channels system corresponds to a three-body system in which one of the three pairs develops a zero-energy bound state.

The behavior we are going to discuss is therefore interesting on its own and also might play a key role in understanding those observed candidates for the exotic hadrons lying in the energy regions where two-body and three-body hadronic thresholds rest close to each other. In this article, we investigate and answer those questions mentioned above.

In Sec. II, we present basic setups, namely, the Hamiltonian we consider, effective interactions constructed by the Feshbach projection, the Alt-Grassberger-Sandhas (AGS) equations which three-body transition amplitudes satisfy, a problem of unphysical singularity, and its solution with the mass renormalization plus an appropriate reorganization of the Feynman diagrams. In Sec. III, we calculate the $S$-matrix pole behavior near the thresholds in a degenerate two-body and three-body coupled-channels system using Yamaguchitype separable interactions. We show that the $S$-matrix pole behavior is characteristic in the system and also universal in a sense that it is determined by the equation $c+$ $E \log (-E)=0$ or, equivalently, $c+\operatorname{Re} E \log (\operatorname{Re} E)=0$ and $\operatorname{Im} E=\pi \operatorname{Re} E / \log (\operatorname{Re} E)$, where $E$ is the $S$-matrix pole energy, while $c$ a real parameter. In Sec. IV, we summarize the results and discuss their physical applications.

\section{EFFECTIVE AGS EQUATIONS FOR TWO-BODY AND THREE-BODY COUPLED-CHANNELS SYSTEM}

We consider a two-body and three-body coupled-channels system. We denote three particles in a three-body channel as $\phi_{1} \phi_{2} \phi_{3}$ and two particles in a two-body channel as $\psi \phi_{3}$. Since we investigate the $S$-matrix pole behavior of a two-body and three-body coupled-channels system by solving the effective three-body system, we first explain how we solve the threebody system with an effective Hamiltonian,

$$
H_{\mathrm{eff}}^{(3)}=\sum_{i=1}^{3}\left(m_{i}+\frac{k_{i}^{2}}{2 m_{i}}\right)+U_{1}+U_{2}+U_{3}+U_{4},
$$

where $U_{1}, U_{2}$, and $U_{3}$ are the effective two-body interactions of $\phi_{2} \phi_{3}, \phi_{3} \phi_{1}$, and $\phi_{1} \phi_{2}$, respectively, and $U_{4}$ is the effective three-body interaction of $\phi_{1} \phi_{2} \phi_{3}$.

The three-body problem can be solved by the following AGS equations $[49,50]$ :

$$
X=Z+Z T X
$$

where the quantities are $3 \times 3$ matrices whose rows and columns correspond to channel states. $X$ is the transition amplitude matrix, $T$ is the diagonal matrix,

$$
T=\left(\begin{array}{ccc}
t_{1} & 0 & 0 \\
0 & t_{2} & 0 \\
0 & 0 & t_{3}
\end{array}\right)
$$

whose diagonal matrix elements $t_{1}, t_{2}$, and $t_{3}$ are the two-body $T$-matrices of $\phi_{2} \phi_{3}, \phi_{3} \phi_{1}$, and $\phi_{1} \phi_{2}$, respectively,

$$
\begin{aligned}
t_{i} & =U_{i}+U_{i} G_{0}^{\phi_{j} \phi_{k}} U_{i}+\cdots \\
& =U_{i} \frac{1}{1-G_{0}^{\phi_{j} \phi_{k}} U_{i}} \quad(i=1,2,3),
\end{aligned}
$$

and $G_{0}^{\phi_{j} \phi_{k}}$ is the free Green function of $\phi_{j} \phi_{k}$,

$$
G_{0}^{\phi_{j} \phi_{k}}=\frac{1}{E-m_{j}-\frac{k_{j}^{2}}{2 m_{j}}-m_{k}-\frac{k_{k}^{2}}{2 m_{k}}} .
$$

$Z$ is composed of two parts:

$$
Z=Z_{0}+Z_{4}
$$

$Z_{0}$ has only off-diagonal elements,

$$
Z_{0}=G_{0}^{\phi \phi \phi} \bar{\delta}
$$

where

$$
\bar{\delta}=\left(\begin{array}{lll}
0 & 1 & 1 \\
1 & 0 & 1 \\
1 & 1 & 0
\end{array}\right),
$$

and $G_{0}^{\phi \phi \phi}$ is the free Green function of $\phi_{1} \phi_{2} \phi_{3}$,

$$
G_{0}^{\phi \phi \phi}=\frac{1}{E-\sum_{i=1}^{3}\left(m_{i}+\frac{k_{i}^{2}}{2 m_{i}}\right)} .
$$

$Z_{4}$ has all $3 \times 3$ components and is a sum of repeated effective three-body force.

The off-diagonal structure of $Z_{0}$ combined with diagonal nature of $T$ prevents overcounting the same two-body $T$ matrices in a row. A diagrammatic representation of the scattering equations is given in Fig. 1. The diagrammatic representation clearly shows what is done in the scattering equations. We first sum a three-body interaction in addition to three two-body interactions to give the three two-body $T$-matrices, $t_{i}(i=1,2,3)$ and $Z_{4}$. We then sum them up, mixing them with each other while taking care of overcounting the same two-body $T$-matrices in a row. The solution of the AGS equations is formally given as

$$
X(E)=\frac{1}{1-Z(E) T(E)} Z(E),
$$




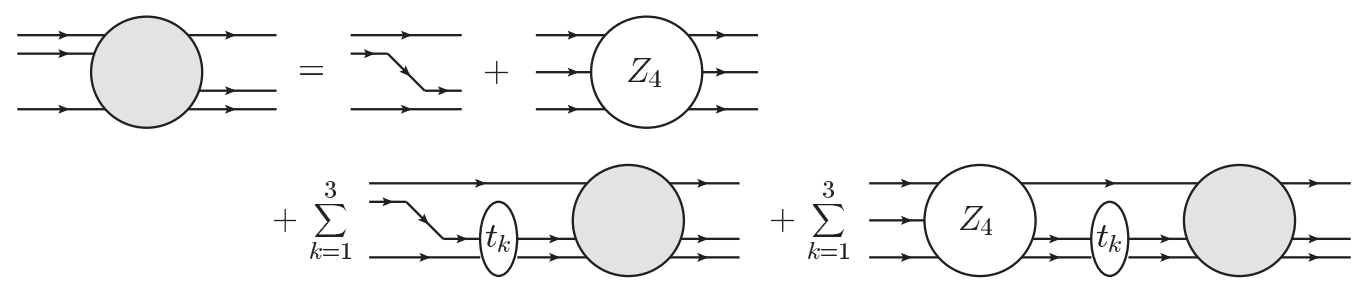

FIG. 1. A diagrammatic representation of the AGS equations with three-body force.

where $E$ is the energy of the three-body system. Considering the eigenvalue equation of the kernel of the AGS equations,

$$
Z(E) T(E)|n\rangle=\eta_{n}(E)|n\rangle,
$$

with the eigenvector and eigenvalue, the formal solution is written as

$$
X(E)=\sum_{n} \frac{|n\rangle\langle n|}{1-\eta_{n}(E)} Z(E) .
$$

If it has an eigenvalue, 1 , at the energy $E=E_{p}$, i.e., $\eta_{n}\left(E_{p}\right)=$ 1, then $X(E)$ has a pole at the energy $E=E_{p}$ as can be seen from Eq. (12). Therefore, we solve the eigenvalue equation of the kernel of the effective AGS equations instead of solving the equations themselves.

We next discuss the reordering of the transition matrix $X$, which is needed in order to understand the singularity structure of $X$ and $Z$. To that end, we decompose the two-body $T$-matrix as

$$
T=\bar{T}_{3}+T_{3}
$$

where, written in an explicit matrix form,

$$
\bar{T}_{3}=\left(\begin{array}{ccc}
t_{1} & 0 & 0 \\
0 & t_{2} & 0 \\
0 & 0 & 0
\end{array}\right), \quad T_{3}=\left(\begin{array}{ccc}
0 & 0 & 0 \\
0 & 0 & 0 \\
0 & 0 & t_{3}
\end{array}\right)
$$

The transition matrix $X$ can be expanded as

$$
X=Z+Z T Z+\cdots=\frac{1}{1-Z T} Z
$$

Since $Z=Z_{0}+Z_{4}$ and $T=T_{3}+\bar{T}_{3}$, we regard the rhs) of Eq. (15) as a function of $Z_{0}, Z_{4}, T_{3}$, and $\bar{T}_{3}$. We first collect the terms including $Z_{4}$ and $T_{3}$ and define the sum to be $W_{3}$ :

$$
W_{3}=Z_{4}+Z_{4} T_{3} Z_{4}+\cdots=\frac{1}{1-Z_{4} T_{3}} Z_{4} .
$$

We next include $Z_{0}$ and define the sum to be $X_{3}$ :

$$
X_{3}=Z_{0}+Z_{0} T_{3} Z_{0}+\left(1+Z_{0} T_{3}\right) W_{3}\left(T_{3} Z_{0}+1\right),
$$

where use has been made of $T_{3} Z_{0} T_{3}=0$. We finally include $\bar{T}_{3}$, which gives $X$ as

$$
\begin{aligned}
X & =X_{3}+X_{3} \bar{T}_{3} X_{3}+X_{3} \bar{T}_{3} X_{3} \bar{T}_{3} X_{3}+\cdots \\
& =X_{3}+X_{3}\left(\bar{T}_{3}+\bar{T}_{3} \bar{X}_{3} \bar{T}_{3}+\cdots\right) X_{3} \\
& =X_{3}+X_{3}\left(\frac{1}{1-\bar{T}_{3} \bar{X}_{3}} \bar{T}_{3}\right) X_{3}
\end{aligned}
$$

where

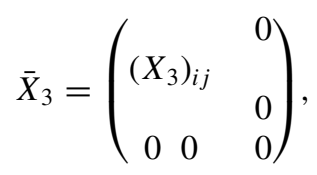

and use has been made of $\bar{T}_{3} X_{3} \bar{T}_{3}=\bar{T}_{3} \bar{X}_{3} \bar{T}_{3}$. Clearly, the three-body pole of $X$ comes from that of $\bar{T}_{3}+\bar{T}_{3} \bar{X}_{3} \bar{T}_{3}+\cdots=$ $\left(1-\bar{T}_{3} \bar{X}_{3}\right)^{-1} \bar{T}_{3}$, which plays an important role in proving the equivalence of the pole in the original and modified AGS equations.

Now, we move on to how we construct effective AGS equations starting from a two-body and three-body coupledchannels system. We first explain what happens if we naively follow the method by Feshbach $[51,52]$. The transition matrix $X$ is known to have the same physical singularities as the original two-body and three-body coupled-channels system. We show, however, that if we try to solve the eigenvalue equation, Eq. (11), we face the problem of an unphysical singularity since a part of the driving term $Z_{4}$ has unphysical singularities. Then, we show that we can define modified AGS equations where not only the transition matrix $X^{\prime}$ but also $Z_{4}^{\prime}$ has only the same physical singularities as the original two-body and three-body coupled-channels system.

We express the effective AGS equations in terms of unrenormalized Green functions in the former argument but in terms of renormalized ones in the latter. This is because the appearance and disappearance of unphysical singularities are most transparent in terms of unrenormalized Green functions in the former argument and in terms of renormalized ones in the latter, respectively. However, even if we express the effective AGS equations in terms of renormalized Green functions in the former or in terms of unrenormalized ones in the latter, the fact that unphysical singularities appear or disappear does not change although the expressions become much more complicated. The origin of the appearance and disappearance of unphysical singularities lies in the reorganization of the higherorder terms of the kernels in the effective AGS equations but not in the use of unrenormalized or renormalized Green functions.

In the method by Feshbach, introducing the projection operators onto the three-body channel, $P$, and the two-body channel, $Q$, respectively, we write the full Hamiltonian as a matrix [51,52]:

$$
H=\left(\begin{array}{cc}
P H P & P H Q \\
Q H P & Q H Q
\end{array}\right)=\left(\begin{array}{cc}
H_{0}^{P}+V_{P P} & V_{P Q} \\
V_{Q P} & H_{0}^{Q}+V_{Q Q}
\end{array}\right) .
$$




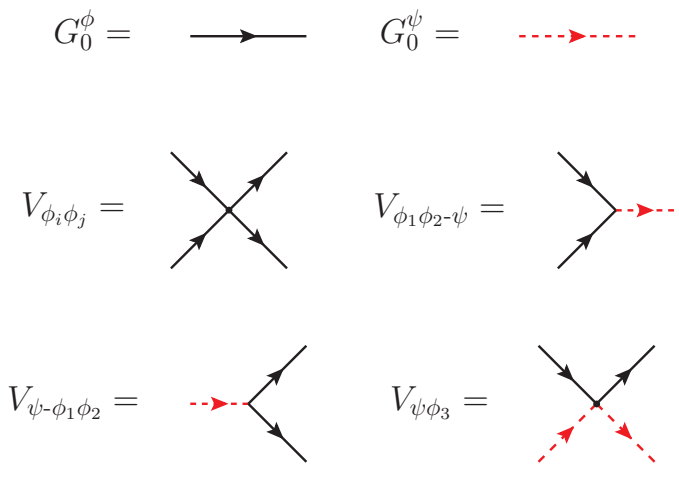

FIG. 2. Summary of Feynman rules. We denote the free Green function of $\phi_{i}$ as solid lines and the unrenormalized free Green function of $\psi$ as dashed red lines.

The kinetic terms in the three-body and two-body channels, $H_{0}^{P}$ and $H_{0}^{Q}$, are respectively given by

$$
\begin{aligned}
& H_{0}^{P}=\sum_{i=1}^{3}\left(m_{i}+\frac{k_{i}^{2}}{2 m_{i}}\right), \\
& H_{0}^{Q}=M+\frac{K_{3}^{2}}{2 M}+m_{3}+\frac{k_{3}^{2}}{2 m_{3}} .
\end{aligned}
$$

We assume that the diagonal interaction terms in the three-body and two-body channels, $V_{P P}$ and $V_{Q Q}$, are given by two-body interactions, while the off-diagonal interaction terms, $V_{P Q}$ and $V_{Q P}$, are due to $\phi_{1} \phi_{2}-\psi$ coupling:

$$
\begin{aligned}
& V_{P P}=V_{\phi_{2} \phi_{3}}+V_{\phi_{3} \phi_{1}}+V_{\phi_{1} \phi_{2}}, \\
& V_{Q Q}=V_{\psi \phi_{3}}, \\
& V_{P Q}=V_{\phi_{1} \phi_{2}-\psi}, \\
& V_{Q P}=V_{\psi-\phi_{1} \phi_{2}} .
\end{aligned}
$$

We define the free Green function of $\phi_{i}, G_{0}^{\phi_{i}}\left(E, k_{i}\right)$, by

$$
G_{0}^{\phi_{i}}\left(E, k_{i}\right)=\frac{1}{E-m_{i}-\frac{k_{i}^{2}}{2 m_{i}}},
$$

and that of $\psi, G_{0}^{\psi}\left(E, K_{3}\right)$, by

$$
G_{0}^{\psi}\left(E, K_{3}\right)=\frac{1}{E-M-\frac{K_{3}^{2}}{2 M}} .
$$

The Feynman rules are therefore given as shown in Fig. 2.

The physical mass of $\psi$ is shifted from the bare one by the coupling to $\phi_{1} \phi_{2}$. The dressed Green function of $\psi, G^{\psi}\left(E, K_{3}\right)$, which is diagrammatically shown as

$$
G^{\psi}=\Longrightarrow
$$

is expressed by the free Green function, $G_{0}^{\psi}\left(E, K_{3}\right)$, and the self-energy, $\Sigma\left(E, K_{3}\right)$, as

$$
\begin{aligned}
G^{\psi}\left(E, K_{3}\right)= & G_{0}^{\psi}\left(E, K_{3}\right)+G_{0}^{\psi}\left(E, K_{3}\right) \Sigma\left(E, K_{3}\right) G_{0}^{\psi}\left(E, K_{3}\right) \\
& +\cdots,
\end{aligned}
$$

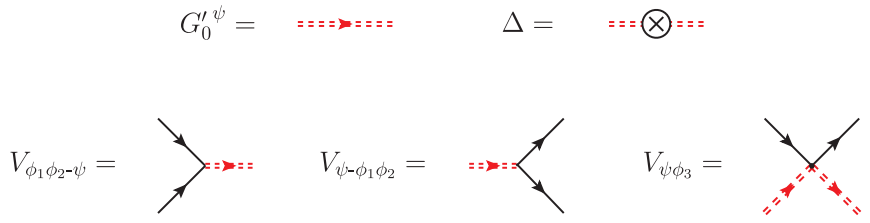

FIG. 3. Summary of Feynman rules. We denote the free Green function of $\phi_{i}$ as solid lines and the unrenormalized free Green function of $\psi$ as dashed red lines.

or diagrammatically,

$$
\Longrightarrow=\cdots \cdots+\cdots-\cdots+\cdots
$$

The physical mass of $\psi, M^{\prime}$, is determined from the pole energy of the Green function with the vanishing momentum, $K_{3}=0$,

$$
M^{\prime}=M+\left.\Sigma(E, 0)\right|_{E=M^{\prime}} .
$$

We also define the renormalized free Hamiltonian as the kinetic term with the physical mass,

$$
H_{0}^{\prime} Q=M^{\prime}+\frac{K^{2}}{2 M^{\prime}}+m_{3}+\frac{k_{3}^{2}}{2 m_{3}},
$$

and add the difference of the bare and renormalized free Hamiltonians, $\Delta$, from the interaction, $V_{\psi \phi_{3}}$ :

$$
\begin{gathered}
H_{0}^{\prime} Q=H_{0}^{Q}-\Delta, \\
V_{\psi \phi_{3}}^{\prime}=V_{\psi \phi_{3}}+\Delta .
\end{gathered}
$$

The renormalized free Green function is defined by

$$
G_{0}^{\prime \psi}\left(E, K_{3}\right)=\frac{1}{E-M^{\prime}-\frac{K_{3}^{2}}{2 M^{\prime}}} .
$$

The Feynman rules in terms of the renormalized free Green function are therefore given as shown in Fig. 3.

In the Feshbach projection method the effective Hamiltonian in the three-body channel is given by

$$
H_{\mathrm{eff}}^{(3)}=P H P+P H Q \frac{1}{E-Q H Q} Q H P=H_{0}^{P}+U_{P P},
$$

where $U_{P P}$ is the effective interaction in the three-body channel:

$$
U_{P P}=V_{P P}+V_{P Q} \frac{1}{E-H_{0}^{Q}-V_{Q Q}} V_{Q P} .
$$

$U_{P P}$ can be decomposed into the sum of the two-body interactions in each channel, $U_{i}(i=1,2,3)$, and the three-body interaction, $U_{4}$ :

$$
U_{P P}=U_{1}+U_{2}+U_{3}+U_{4},
$$

where

$$
\begin{aligned}
& U_{1}=V_{\phi_{2} \phi_{3}}= \\
& U_{2}=V_{\phi_{3} \phi_{1}}=
\end{aligned}
$$


$U_{3}=V_{\phi_{1} \phi_{2}}+V_{\phi_{1} \phi_{2}-\psi} G_{0}^{\psi \phi_{3}} V_{\psi-\phi_{1} \phi_{2}}=\stackrel{\phi_{1}}{\phi_{2}}$

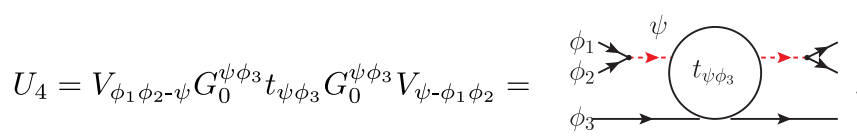

and

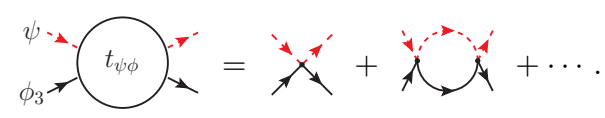

The matrix, $Z_{4}$, is channel independent, i.e., $Z_{4}=z_{4} \mathbf{1}$, where

$$
\mathbf{1}=\left(\begin{array}{lll}
1 & 1 & 1 \\
1 & 1 & 1 \\
1 & 1 & 1
\end{array}\right)
$$

$z_{4}$ is given by

$$
z_{4}=G_{0}^{\phi \phi \phi} U_{4} G_{0}^{\phi \phi \phi}+\cdots=G_{0}^{\phi \phi \phi} U_{4} \frac{1}{1-G_{0}^{\phi \phi \phi} U_{4}} G_{0}^{\phi \phi \phi}
$$

or diagrammatically,

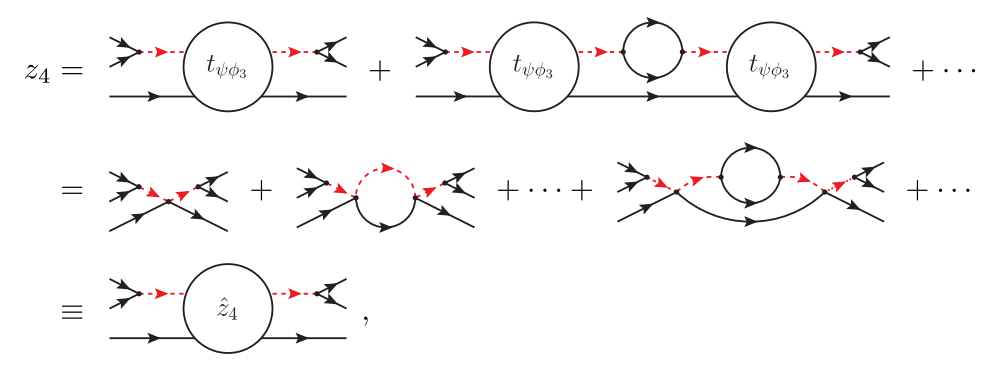

and $z_{4} t_{3} z_{4}$ by

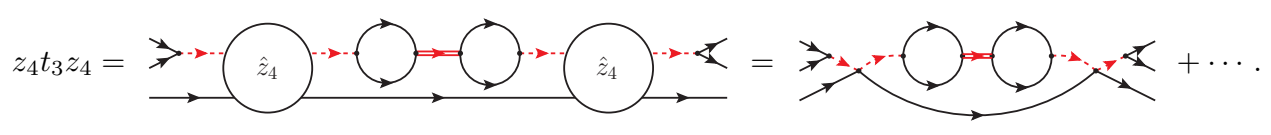

The matrix, $W_{3}$, is also channel independent, i.e. $W_{3}=w_{3} \mathbf{1}$, and $w_{3}=z_{4}+z_{4} t_{3} z_{4}+\cdots$ is diagrammatically given by

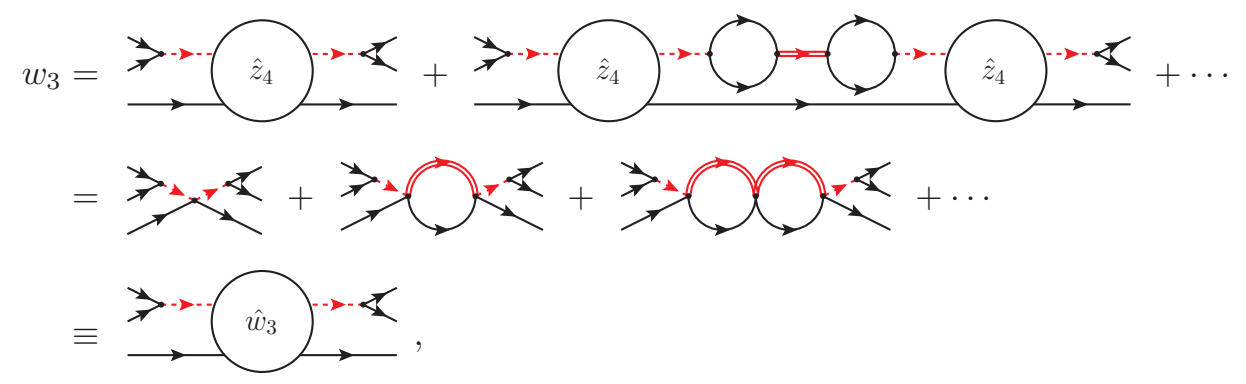

where in the intermediate state use has been made of the relation for the dressed and free Green functions for $\psi$,

$$
G^{\psi}=G_{0}^{\psi}+G_{0}^{\psi} \Sigma G_{0}^{\psi}+G_{0}^{\psi} \Sigma G^{\psi} \Sigma G_{0}^{\psi}
$$

or diagrammatically,

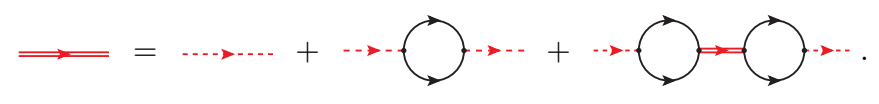

The first two terms in Eq. (47) or Eq. (48), $G_{0}^{\psi}$ and $G_{0}^{\psi} \Sigma G_{0}^{\psi}$, are included in $Z_{4}\left(z_{4}\right)$, while the last term $G_{0}^{\psi} \Sigma G^{\psi} \Sigma G_{0}^{\psi}$ in included in $Z_{4} T_{3} Z_{4}\left(z_{4} t_{3} z_{4}\right)$, and the sum of these terms gives the full Green function, $G^{\psi}$, in $W_{3}\left(w_{3}\right)$. Thus, it is clear that $Z_{4}$, and therefore $Z$, has an unphysical branch point at $E=M+m_{3}$, while $W_{3}$, and therefore $X$, has only a physical one at $E=M^{\prime}+m_{3}$. If we solve for the scattering amplitudes $X$, this unphysical singularity of $Z$ does not matter. However, since we solve the eigenvalue equation of $Z T$, which has an unphysical singularity, the existence of the unphysical singularity causes difficulty in searching for the $S$-matrix pole in the complex energy plane.

Later, we modify the effective AGS equations and compare the matrix element, $\bar{X}_{3}$, in the original and modified effective AGS equations. Therefore, we show here the $i j(i, j=1$ or 2$)$ matrix element of the third term of Eq. $(17),\left(1+Z_{0} T_{3}\right) W_{3}\left(T_{3} Z_{0}+1\right)$, 
which is given as

$$
\left(\left(1+Z_{0} T_{3}\right) W_{3}\left(T_{3} Z_{0}+1\right)\right)_{i j}=\left(1+G_{0}^{\phi \phi \phi} t_{3}\right) w_{3}\left(1+t_{3} G_{0}^{\phi \phi \phi}\right)
$$

where use has been made of the relation

$$
G^{\psi}=G_{0}^{\psi}+G_{0}^{\psi} \Sigma G^{\psi}
$$

or diagrammatically,

$$
\Longrightarrow=\cdots \cdots+\cdots-\longrightarrow
$$

Let us now discuss the modification of the effective AGS equations. Our task is to find the modified AGS equations

$$
\begin{aligned}
& X^{\prime}(E)=Z^{\prime}(E)+Z^{\prime}(E) T(E) X^{\prime}(E), \\
& Z^{\prime}(E)=Z_{0}(E)+Z_{4}^{\prime}(E),
\end{aligned}
$$

where the modified transition amplitude, $X^{\prime}$, has the same three-body pole as the original amplitude, $X$, yet the modified kernel, $Z^{\prime}$, has only physical singularities.

We first define $t_{\psi \phi_{3}}^{\prime}$ by summing up the higher-order terms of $t_{\psi \phi_{3}}$, which includes counterterms, as

$$
\Rightarrow===t_{\psi \phi_{3}}^{\prime}=\Rightarrow==
$$

Then, we define $z_{4}^{\prime}$ similarly to Eq. (44), which is diagrammatically given by

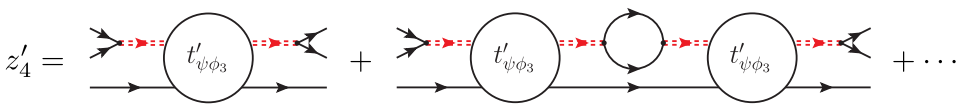

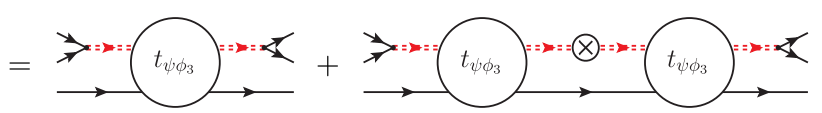

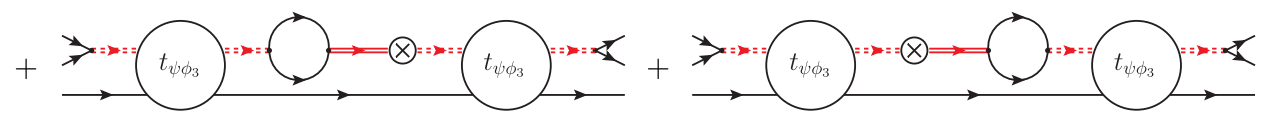

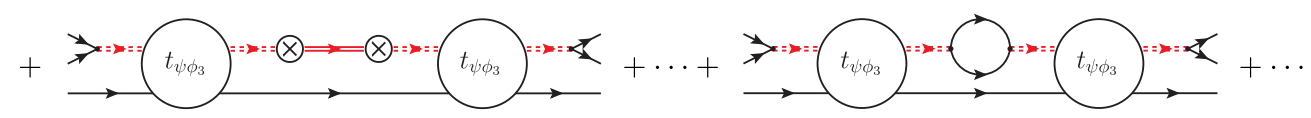

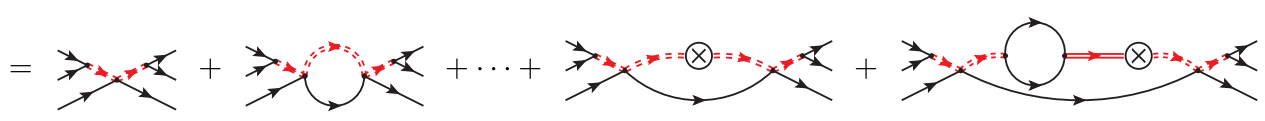

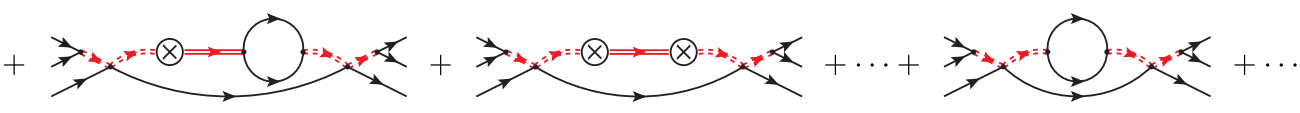

$$
\begin{aligned}
& \equiv \underset{\hat{z}_{4}^{\prime}}{\longrightarrow}=:=<<
\end{aligned}
$$


and $z_{4}^{\prime} t_{3} z_{4}^{\prime}$ by

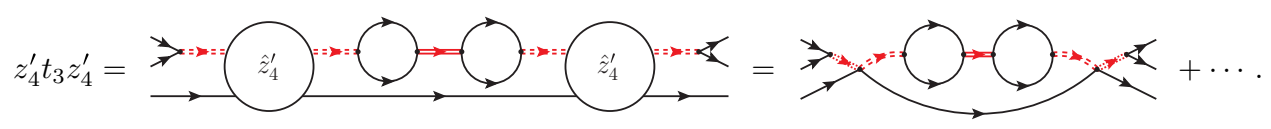

Recalling that the corresponding counterterms of $z_{4}^{\prime} t_{3} z_{4}^{\prime}$ are included in $z_{4}^{\prime}$, we can show that $w_{3}^{\prime}=z_{4}^{\prime}+z_{4}^{\prime} t_{3} z_{4}^{\prime}+\cdots$ is given by

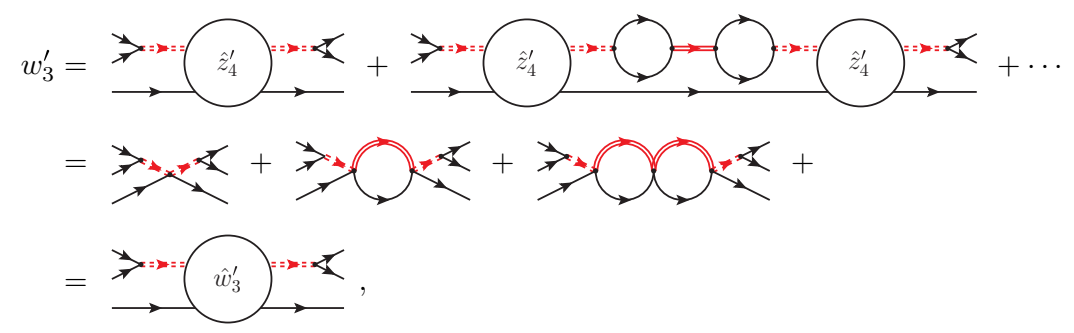

where use has been made of the relation for the dressed and renormalized free Green functions for $\psi$,

$$
\begin{aligned}
G^{\psi} & =G_{0}^{\prime \psi}+G_{0}^{\prime \psi}(\Sigma+\Delta) G_{0}^{\prime \psi}+G_{0}^{\prime \psi}(\Sigma+\Delta) G^{\psi}(\Sigma+\Delta) G_{0}^{\prime \psi} \\
& =G_{0}^{\prime \psi}+G_{0}^{\prime \psi} \Sigma G_{0}^{\prime \psi}+G_{0}^{\prime \psi} \Delta G_{0}^{\prime \psi}+G_{0}^{\prime \psi} \Delta G^{\psi} \Delta G_{0}^{\prime \psi}+G_{0}^{\prime \psi} \Delta G^{\psi} \Sigma G_{0}^{\prime \psi}+G_{0}^{\prime \psi} \Sigma G^{\psi} \Delta G_{0}^{\prime \psi}+G_{0}^{\prime \psi} \Sigma G^{\psi} \Sigma G_{0}^{\prime \psi},
\end{aligned}
$$

or diagrammatically,

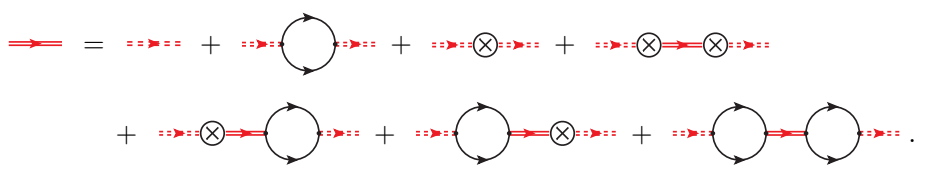

Clearly, the vertex $\hat{w}_{3}^{\prime}$ is the same as $\hat{w}_{3}$, yet, given as a finite sum of the renormalized free Green function, $z_{4}^{\prime}$ has a physical branch point at $E=M^{\prime}+m_{3}$, not an unphysical one at $E=M+m_{3}$ in contrast to $z_{4}$. This is because we define $z_{4}^{\prime}$ as a finite sum of the renormalized free Green function, and therefore it includes not only $z_{4}$ but also a part of the higher-order terms, $z_{4} t_{3} z_{4}, z_{4} t_{3} z_{4} t_{3} z_{4}$, and so on, which changed the bare singularity to the physical one.

In contrast to $Z_{4}$, we define the matrix $Z_{4}^{\prime}$ not to be proportional to 1 but to be given as (see Appendix A for details)
The matrix element of $W_{3}^{\prime}$, which is defined by $W_{3}^{\prime}=Z_{4}^{\prime}+$ $Z_{4}^{\prime} T_{3} Z_{4}^{\prime}+Z_{4}^{\prime} T_{3} Z_{4}^{\prime} T_{3} Z_{4}^{\prime}+\cdots$, is given as

$$
\begin{aligned}
\left(W_{3}^{\prime}\right)_{\alpha \beta} & =\left(Z_{4}^{\prime}\right)_{\alpha \beta}+\left(Z_{4}^{\prime}\right)_{\alpha 3} t_{3}\left(Z_{4}^{\prime}\right)_{3 \beta}+\left(Z_{4}^{\prime}\right)_{\alpha 3} t_{3} z_{4}^{\prime} t_{3}\left(Z_{4}^{\prime}\right)_{3 \beta}+\cdots \\
& =\left(Z_{4}^{\prime}\right)_{\alpha 3} \frac{z_{4}^{\prime}+z_{4}^{\prime} t_{3} z_{4}^{\prime}+z_{4}^{\prime} t_{3} z_{4}^{\prime} t_{3} z_{4}^{\prime}+\cdots}{z_{4}^{\prime 2}}\left(Z_{4}^{\prime}\right)_{3 \beta} \\
& =\left(Z_{4}^{\prime}\right)_{\alpha 3} \frac{w_{3}^{\prime}}{z_{4}^{\prime 2}}\left(Z_{4}^{\prime}\right)_{3 \beta} .
\end{aligned}
$$

Therefore, $W_{3}^{\prime}$ has a matrix structure similar to $Z_{4}^{\prime}$ as

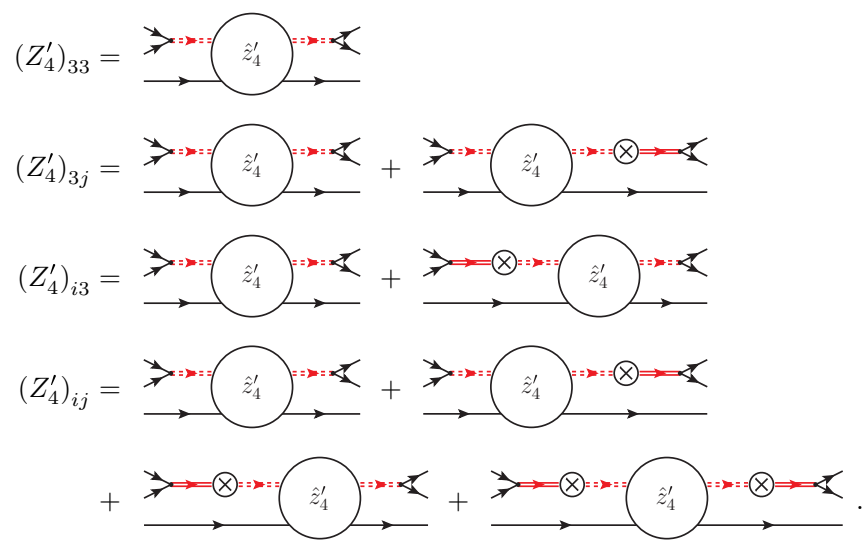

(60)

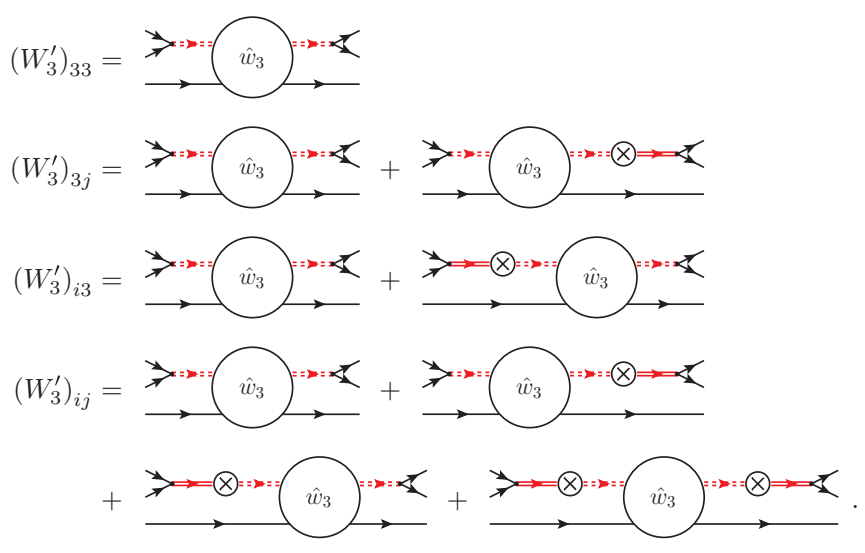

(62) 
Then, the $i j(i, j=1$ or 2$)$ matrix element of the third term of Eq. (17), $\left(1+Z_{0} T_{3}\right) W_{3}^{\prime}\left(T_{3} Z_{0}+1\right)$, is given as

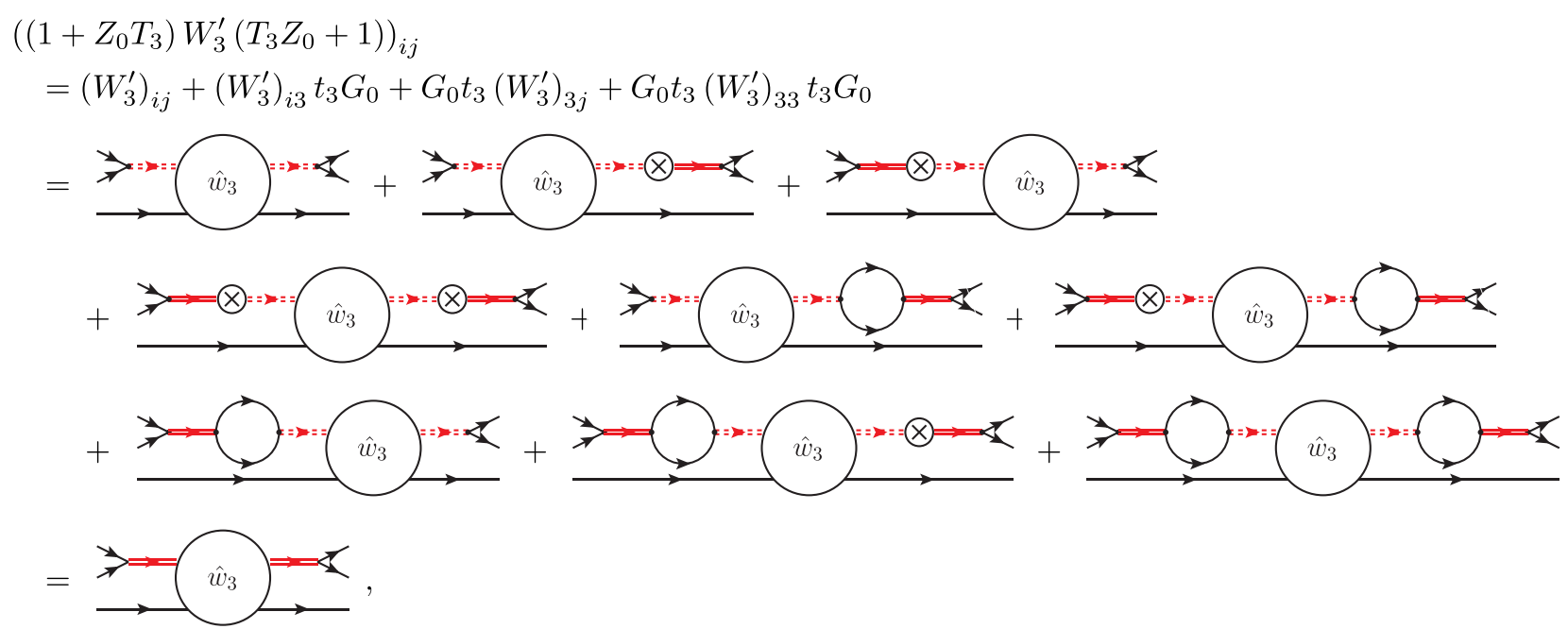

where use has been made of the relation

$$
G^{\psi}=G_{0}^{\prime \psi}+G_{0}^{\prime \psi} \Sigma G^{\psi}+G_{0}^{\prime \psi} \Delta G^{\psi}
$$

or, diagrammatically,

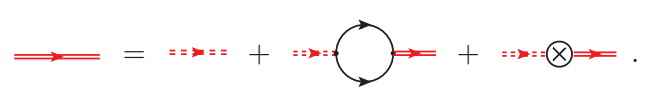

From Eqs. (49) and (63) one sees that

$$
\left(\left(1+Z_{0} T_{3}\right) W_{3}^{\prime}\left(T_{3} Z_{0}+1\right)\right)_{i j}=\left(\left(1+Z_{0} T_{3}\right) W_{3}\left(T_{3} Z_{0}+1\right)\right)_{i j} .
$$

The first and second terms of Eq. (17), $Z_{0}$ and $Z_{0} T_{3} Z_{0}$, are also the same in the original and modified equations, since we have modified neither $Z_{0}$ nor $T_{3}$. Therefore,

$$
\left(X_{3}^{\prime}\right)_{i j}=\left(X_{3}\right)_{i j}
$$

and

$$
\frac{\bar{T}_{3}}{1-\bar{T}_{3} \bar{X}_{3}^{\prime}}=\frac{\bar{T}_{3}}{1-\bar{T}_{3} \bar{X}_{3}},
$$

from which we conclude that the modified transition amplitude, $X^{\prime}$, has the same three-body pole as the original amplitude, $X$.

\section{THE $S$-MATRIX POLE BEHAVIOR NEAR THE THRESHOLD: NUMERICAL RESULTS}

In this section, we numerically solve the eigenvalue equation of the kernel of the effective AGS equations and analyze the $S$-matrix pole behavior near the thresholds in the case of a degenerate two-body and three-body coupled-channels system.

We adopt separable interactions both for diagonal and offdiagonal parts whose matrix elements are

$$
\begin{aligned}
\left\langle k_{i} k_{j}\left|V_{\phi_{i} \phi_{j}}\right| k_{i}^{\prime} k_{j}^{\prime}\right\rangle & =g\left(p_{k}\right) \lambda_{\phi_{i} \phi_{j}} g\left(p_{k}^{\prime}\right), \\
\left\langle K k_{3}\left|V_{\psi \phi_{3}}\right| K^{\prime} k_{3}^{\prime}\right\rangle & =g\left(q_{3}\right) \lambda_{\psi \phi} g\left(q_{3}^{\prime}\right), \\
\left\langle K\left|V_{\psi-\phi_{1} \phi_{2}}\right| k_{1} k_{2}\right\rangle & =\frac{1}{K^{2}} \delta\left(K-k_{1}-k_{2}\right) \lambda_{\psi-\phi \phi} g\left(p_{3}\right),
\end{aligned}
$$

where $p_{k}$ is the relative momentum of $\phi_{i}$ and $\phi_{j}, p_{k}=\left(k_{i}-\right.$ $\left.k_{j}\right) / 2$, while $q_{3}$ is that of $\psi$ and $\phi_{3}, q_{k}=\left(K-k_{3}\right) / 2 . g(p)$ is the Yamaguchi-type form factor $g(p)=\Lambda^{2} /\left(p^{2}+\Lambda^{2}\right)$, where $\Lambda$ is called a cutoff parameter. $\lambda_{\phi \phi}$ and $\lambda_{\psi \phi}$ are the coupling constants of the two-body interactions in the two-body and three-body channels, respectively. In the two-body channel we take the coupling constants of the two-body interactions of $\phi_{2} \phi_{3}$ and $\phi_{3} \phi_{1}$ to be the same, $\lambda_{\phi \phi}$, but that of $\phi_{1} \phi_{2}$ to be zero. $\lambda_{\psi-\phi \phi}$ is the $\psi-\phi_{1} \phi_{2}$ coupling constant.

Bound-state poles lie on the real physical energy axis below the threshold, which can be directly studied by the eigenvalue equation of the kernel of the modified effective AGS equations

$$
Z^{\prime}(E) T(E)|n\rangle=\eta_{n}(E)|n\rangle .
$$

However, resonance poles are located in the fourth quadrant of the unphysical energy sheet, ${ }^{1}$ which can be exposed only after analytic continuation of the eigenvalue equation [Eq. (72)] is performed onto the unphysical complex energy sheet. For this purpose we adopt the method of contour rotation. See, for example, Refs. [54,55].

The contour rotation is done by rotating the integration contour as follows:

$$
\begin{aligned}
& \sum_{j=1}^{3} \int_{0}^{\infty} q_{j}^{2} d q_{j} Z^{\prime}\left(E q_{i} q_{j}\right) \tau_{j}\left(E-m_{j}-\frac{q_{j}^{2}}{2 M_{j}}\right) \phi\left(q_{j}\right) \\
& \quad=\eta(E) \phi\left(q_{i}\right),
\end{aligned}
$$

\footnotetext{
${ }^{1}$ It is known that the two-body channel has a square root singularity, $\left(\Delta_{2}-E\right)^{1 / 2}$, and the three-body channel has a logarithmic singularity, $\left(E-\Delta_{3}\right)^{2} \log \left(\Delta_{3}-E\right)$ [53], where we take the two-body threshold energy, $\Delta_{2}$, and the three-body threshold energy, $\Delta_{3}$, independently for explanation. We consider the complex energy sheet with $\pi / 2<$ $\arg \left(\Delta_{2}-E\right)^{1 / 2}<3 \pi / 2$ and $\pi<\operatorname{Im} \log \left(\Delta_{3}-E\right)<3 \pi$, which is directly connected to the real physical energy $\arg \left(\Delta_{2}-E\right)^{1 / 2}=\pi / 2$ and $\operatorname{Im} \log \left(\Delta_{3}-E\right)=\pi$, by rotating the energy clockwise and we simply refer to it as the "unphysical energy sheet."
} 


$$
\begin{aligned}
\Rightarrow & \sum_{j=1}^{3} \int_{0}^{\infty} e^{-3 i \theta} q_{j}^{2} d q_{j} Z^{\prime}\left(E e^{-i \theta} q_{i} e^{-i \theta} q_{j}\right) \tau_{j} \\
& \times\left(E-m_{j}-\frac{e^{-2 i \theta} q_{j}^{2}}{2 M_{j}}\right) \phi\left(e^{-i \theta} q_{j}\right)=\eta(E) \phi\left(e^{-i \theta} q_{i}\right) .
\end{aligned}
$$

Since we adopt a Yamaguchi-type form factor, the rotation angle $\theta$ in the momentum plane is restricted to $\theta \leqslant \frac{\pi}{2}$ and therefore an angle of rotation of the branch cut in the complex energy sheet is restricted to be less than $\pi$. This causes no problem since we are interested in resonances lying on the fourth quadrant of the unphysical complex energy sheet.

After performing analytical continuation, we twodimensionally discretize the fourth quadrant of the complex energy sheet with the interval 0.00025 and calculate the Fredholm determinant, $\prod_{n}\left(1-\eta_{n}(E)\right)$, of the kernel, $Z(E) T(E)$, at each grid. We then identify the complex energy, at which the Fredholm determinant takes a minimum, as an approximate pole energy, regarding discretization intervals as errors.

Here we summarize how we take the parameters of the model. We assume the masses of $\phi_{i}$ to be the same and take them as the unit of the energy, $m_{1}=m_{2}=m_{3}=1$. The physical mass of $\psi$ is therefore $M^{\prime}=2$ in our unit. It is useful to define dimensionless coupling constants $f_{\phi \phi}$ and $f_{\psi \phi}$ as $f_{\phi \phi}=\frac{\pi}{2} \mu_{\phi \phi} \lambda_{\phi \phi} \Lambda$ and $f_{\psi \phi}=\frac{\pi}{2} \mu_{\psi \phi} \lambda_{\psi \phi} \Lambda$, where $\mu_{\phi \phi}$ and $\mu_{\psi \phi}$ are reduced masses of $\phi \phi$ and $\psi \phi$, respectively. When $f_{\phi \phi} \leqslant-1\left(f_{\psi \phi} \leqslant-1\right)$ the two-body system, $\phi_{2} \phi_{3}$ or $\phi_{3} \phi_{1}$ $\left(\psi \phi_{3}\right)$ has a bound state. Matrix elements of the effective interactions, two-body and three-body $T$-matrices, and driving terms are presented in Appendix B. We fix the off-diagonal coupling constant, $\lambda_{\psi-\phi \phi}$, to be $\sqrt{4 \pi} \times 0.1$ in our unit for simplicity, while we change the diagonal coupling constants; i.e., we take 6 values, $-0.15,-0.2,-0.25,-0.3,-0.35$, and -0.4 , for $f_{\phi \phi}$, and 10 to 15 values between -1.11 and -1.41 for $f_{\psi \phi}$.

In Table I, we summarize obtained complex pole energies vs. coupling constants, $f_{\phi \phi}$ and $f_{\psi \phi}$. Then, in Fig. 4 (Fig. 5), we plot locations of poles in the unphysical complex energy sheet with error bars explained above for different values of $f_{\psi \phi}$ $\left(f_{\phi \phi}\right)$ with $f_{\phi \phi}\left(f_{\psi \phi}\right)$ fixed. We also show the curve determined by the equation

$$
c+E \log (-E)=0,
$$

where $c$ is a real parameter, which corresponds to the inverse scattering length in the $s$-wave two-body scattering. When the pole is located in the fourth quadrant of the unphysical complex energy plane, the real and imaginary parts of Eq. (74), respectively, become

$$
c+\operatorname{Re} E \log (\operatorname{Re} E)=0, \quad \operatorname{Im} E=\pi \operatorname{Re} E / \log (\operatorname{Re} E),
$$

where higher-order terms of $\operatorname{Im} E / \operatorname{Re} E$ are neglected.

In Figs. 4 and 5 one sees that the calculated results are consistent with the analytic formula, Eqs. (74) or (75), near the degenerate threshold within error bars. In Figs. 4 and 5 the trajectories of poles seem slightly unsmooth. This might be due to the slow convergence of the integral equation when poles lie close to the rotated branch cut. However, the deviations are still within errors.

We see that the $S$-matrix pole approaches the threshold from the fourth quadrant of the unphysical complex energy sheet as the absolute value of the coupling constant, $f_{\psi \phi}$ $\left(f_{\psi \psi}\right)$, increases. We also see that when poles lie close to the threshold, that is, when $\operatorname{Re} E \lesssim 0.001$, they all lie on the curve, $c+E \log (-E)=0$. This is consistent with the analytical study of Ref. [56]. The $S$-matrix pole behavior near the threshold in the system is therefore universal in the sense that it is described by the unique one-parameter equation $c+E \log (-E)=0$ irrespective of details of the specific parameter sets.

This behavior of the $S$-matrix pole near the threshold is different from either the two-body or three-body scattering. In the two-body scattering the $T$-matrix near the threshold is represented in the form of the effective range expansion and the pole is given as a solution of the equation

$$
-\frac{1}{a_{\ell}}+\frac{r_{\ell}}{2} p^{2}+\cdots-i p^{2 \ell+1}=0 \quad\left(E=\frac{p^{2}}{2 \mu}\right),
$$

where $\ell$ is the relative angular momentum, and $p$ and $\mu$ are the relative momentum and the reduced mass of the two particles, respectively. From Eq. (76) the behavior of the pole momentum in the unphysical complex energy sheet is given as

$$
\begin{aligned}
p & \sim-i / a_{0}(\ell=0), \\
p & \sim \pm \sqrt{\frac{2}{a_{\ell} r_{\ell}}}-i \frac{2^{\ell}}{a_{\ell}^{\ell} r_{\ell}^{\ell+1}} \quad(\ell \geqslant 1) .
\end{aligned}
$$

If $\ell=0$, the $S$-matrix pole approaches the threshold from the negative axis in the unphysical complex energy sheet and becomes a bound state as the interaction becomes more attractive. If $\ell \geqslant 1$, the pole approaches the threshold from the fourth quadrant of the unphysical complex energy sheet, which manifests itself as a resonance if it lies close enough to the real axis, and becomes a bound state as the interaction becomes more attractive. Figures 6 and 7 illustrate trajectories of poles of $\ell=0$ and $\ell \geqslant 1$, respectively, both in the physical and in the unphysical complex energy sheet. ${ }^{2}$

In the three-body scattering, the $T$-matrix near the threshold behaves as in the two-body scattering with $\ell$ replaced by $\mathcal{L}=$ $L+\frac{3}{2}$, where $L$ is the sum of two relative angular momenta in the three-body system [48]. Therefore, the behavior of the $S$-matrix pole in the three-body scattering is similar to that of the two-body scattering with $\ell \geqslant 1$ and resonances can exist irrespective of the relative angular momenta.

It should be noted that in the degenerate two-body and threebody coupled-channels system the $S$-matrix pole approaches the degenerate threshold from the fourth quadrant of the

\footnotetext{
${ }^{2}$ The above argument implicitly assumes that the energy dependence of the interaction is not strong. If the energy dependence of the interaction is very strong, even the two-body system with $\ell=0$ can have resonances. See, for instance, Ref. [57].
} 


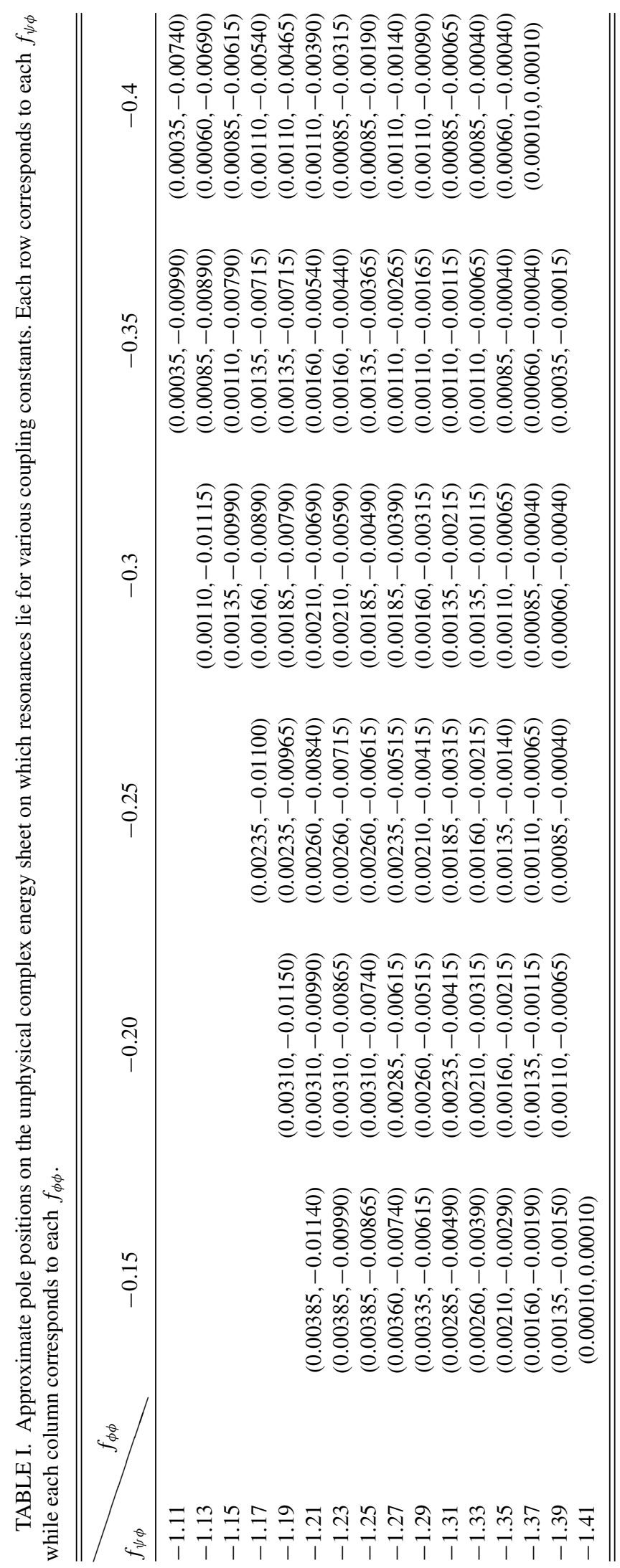



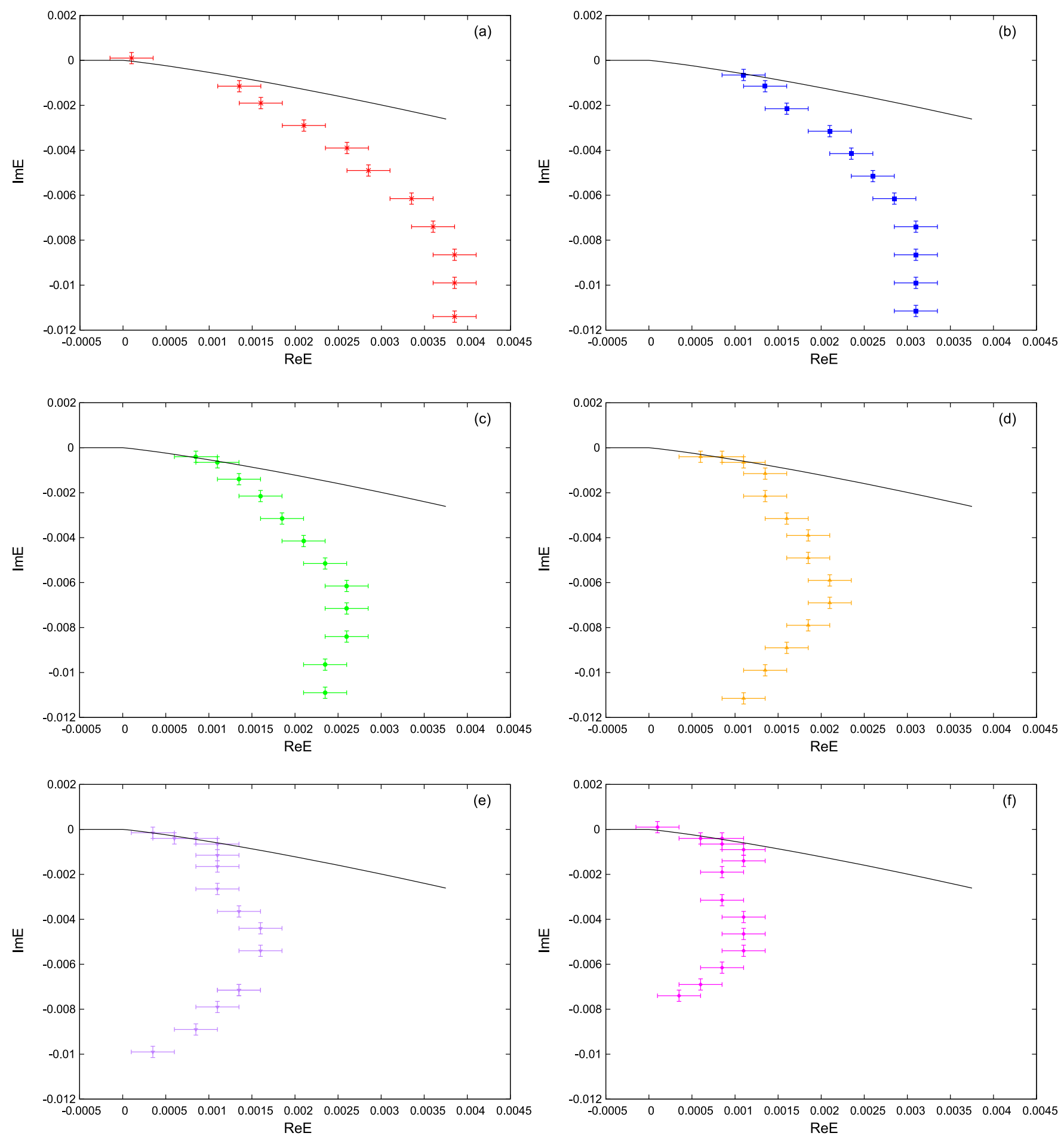

FIG. 4. The $S$-matrix pole trajectories near the thresholds in a degenerate two-body and three-body coupled-channels system: (a) $f_{\phi \phi}=$ -0.15 , (b) -0.20 , (c) -0.25 , (d) -0.30 , (e) -0.35 , and (f) -0.40 . Each point in each figure corresponds to different $f_{\psi \phi}$. The solid curve is a trajectory determined by the equation $c+E \log (-E)=0$, or equivalently $c+\operatorname{Re} E \log (\operatorname{Re} E)=0$ and $\operatorname{Im} E=\pi \operatorname{Re} E / \log (\operatorname{Re} E)$. We can see that poles approach the thresholds from the fourth quadrant of the unphysical complex energy sheet. We can also see that when poles lie very close to the thresholds, they all lie on the solid curve irrespective of parameter sets.

unphysical complex energy sheet even though the two-body system is in the $s$ wave, which is different from that of the two-body $s$-wave scattering. Therefore, if poles lie close enough to the real axis, they might appear as resonances.

\section{SUMMARY AND DISCUSSION}

In this paper, we studied the $S$-matrix pole behavior near the threshold for the degenerate two-body and threebody coupled-channels system. To that end, we formulated 

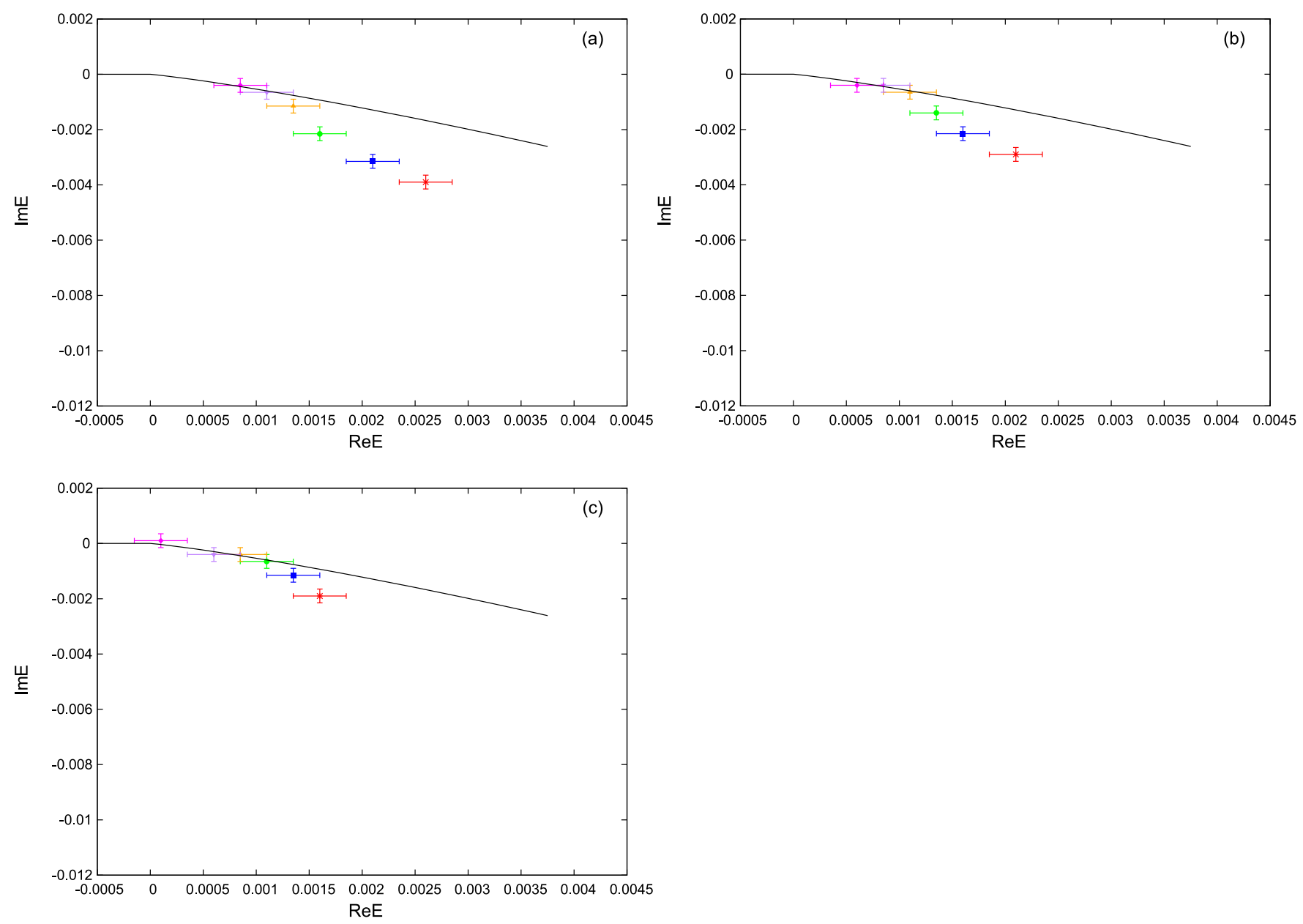

FIG. 5. The $S$-matrix pole trajectories near the thresholds in a degenerate two-body and three-body coupled-channels system: (a) $f_{\psi \phi}=$ -1.33 , (b) -1.35 , and (c) -1.37 . Each point in each figure corresponds to different $f_{\phi \phi}$. The solid curve is a trajectory determined by the equation $c+E \log (-E)=0$, or equivalently $c+\operatorname{Re} E \log (\operatorname{Re} E)=0$ and $\operatorname{Im} E=\pi \operatorname{Re} E / \log (\operatorname{Re} E)$. We can see that poles approach the thresholds from the fourth quadrant of the unphysical complex energy sheet. We can also see that when poles lie very close to the thresholds, they all lie on the solid curve irrespective of parameter sets.

two-body and three-body coupled-channels scattering equations as effective three-body scattering equations, effective AGS equations, by the Feshbach projection method. In the

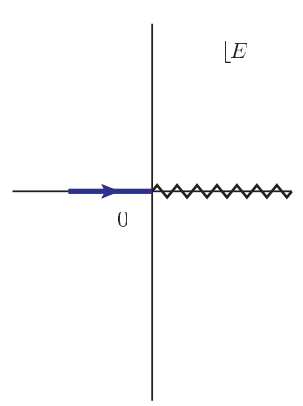

(a)

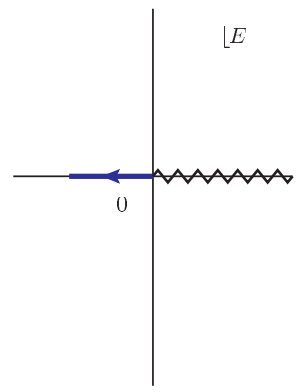

(b)
FIG. 6. The $S$-matrix pole trajectory: $s$-wave. (a) The unphysical complex energy sheet and (b) The physical complex energy sheet. effective AGS equations, effects induced by the coupling to the two-body channel are embedded as effective interactions in the three-body channel. Even in the absence of elementary

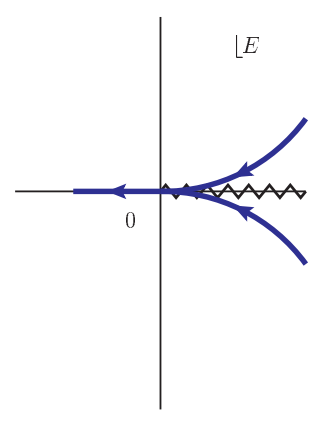

(a)

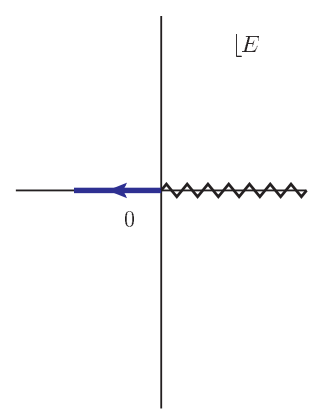

(b)
FIG. 7. The $S$-matrix pole trajectory: higher partial wave. (a) The unphysical complex energy sheet and (b) The physical complex energy sheet. 
three-body interactions, the coupling to the two-body channel generates effective three-body interactions.

We solved the eigenvalue equation of the kernel of the scattering equations instead of solving the equations themselves to obtain the $S$-matrix pole energy. However, we faced the problem of an unphysical singularity when we naively tried to solve the eigenvalue equation. Namely, it turns out that the solution of the eigenvalue equation has an unphysical branch point due to the fact that the physical mass of a particle is different from the bare one. The full transition amplitudes of course have only the physical singularities; i.e., they have a physical branch point with the physical mass but not an unphysical branch point with the bare mass. We showed that this problem is resolved by an appropriate reorganization of the scattering equations and the mass renormalization. In a word, we included the counterterms which appear in higherorder terms of the scattering equations into the kernel of the scattering equations, which is an input of the eigenvalue equation.

We numerically solved the modified eigenvalue equation and obtained the $S$-matrix pole behavior near the thresholds for various parameters. The $S$-matrix pole approaches the threshold from the fourth quadrant of the unphysical energy sheet, which manifests itself as a resonance if it lies close enough to the real axis, and becomes a bound state as the interaction becomes more attractive. The behavior of the obtained numerical results is consistent with the universal behavior $c+E \log (-E)=0$ analytically obtained in Ref. [56]. This behavior is different from the two-body or three-body scatterings and is characteristic in the degenerate two-body and three-body coupled-channels system. This behavior, however, is analogous to the two-body system with non-vanishing angular momentum or the three-body system in the sense that the states appear either as bound states or as resonances but never as virtual states. This generally holds in the degenerate two-body and three-body coupled-channels system independent of the parameters taken in the calculation, which can be interpreted as an appearance of the effective generalized angular momentum barrier as in the three-body system.

In this paper we considered an ideal situation with exactly degenerate two-body and three-body thresholds, which is unrealistic. To what extent would what we have found in the present paper survive when two-body and three-body thresholds are not exactly degenerate? Let $\Delta_{2}$ and $\Delta_{3}$ be the threshold energies of the two-body and three-body channels, respectively, and $E_{p}$ be the resonance energy. If $\left|E_{p}-\min \left\{\Delta_{2}, \Delta_{3}\right\}\right| \gg$ $\left|\Delta_{2}-\Delta_{3}\right|$, then what we have found would survive as a leading order approximation, while if $\left|E_{p}-\min \left\{\Delta_{2}, \Delta_{3}\right\}\right| \ll$ $\left|\Delta_{2}-\Delta_{3}\right|$, then the upper threshold would become irrelevant and the behavior of the resonance would be governed by the lower threshold.

The characteristic behavior predicted in this paper for the degenerate two-body and three-body coupled-channels system may be observed in the cold atom system in which the interaction can be controlled by applying external fields or (approximately) in the hadronic systems where two-body and three-body thresholds are (approximately) degenerate.

\section{ACKNOWLEDGMENTS}

We would like to thank Pascal Naidon for discussions about Efimov physics in general and also useful comments on our work. We would also like to thank Hiroyuki Kamano, Toru Sato, and Koichi Yazaki for helpful discussions. We would also like to thank the computational environment at the High Energy Accelerator Research Organization (KEK). The work of A.K. is partly supported by the University of Tokyo research assistant grants for Ph.D. research and research assistant grants by KEK.

\section{APPENDIX A: MODIFIED KERNEL IN THE PRESENCE OF $V_{\phi_{1} \phi_{2}}$}

In Sec. II, we derived the modified kernel by reorganizing the Feynman diagrams order-by-order with respect to $V_{\psi \phi_{3}}$, the interaction between $\psi \phi_{3}$. There, we ignored the elementary interaction between $\phi_{1} \phi_{2}$ denoted as $V_{\phi_{1} \phi_{2}}$ to simplify the argument. In this Appendix, we present a detailed derivation of the modified kernel taking also $V_{\phi_{1} \phi_{2}}$ into account and by explicitly summing each term in the effective AGS equations as a geometric series. In the following, we suppress the argument of energy $E$ for notational simplicity.

\section{The self-energies in higher-order terms in the effective AGS equations}

In this section, we see how the self-energies appear in higher-order terms of the effective AGS equations and how the corresponding counterterms are added to it. Derivation of the modified kernel is given in the next section.

In the presence of $V_{\phi_{1} \phi_{2}}$, the self-energy can be decomposed into two parts, one that contains the $\phi_{1} \phi_{2}$ interaction, which we denote as $\Sigma_{V}$, and the other that does not, denoted as $\Sigma_{0}$ :

$$
\Sigma=\Sigma_{0}+\Sigma_{V}
$$

Its diagrammatic representation is given as

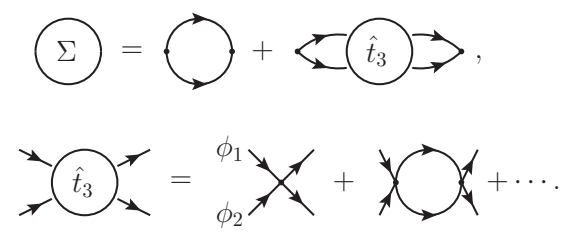

When it comes to considering the self-energies which appear in higher-order terms of the effective AGS equations, it is 
convenient to write $t_{3}$ in the following form:

$$
t_{3}=\hat{t}_{3}+\left(1+\hat{t}_{3} G_{0}^{\phi \phi \phi}\right) V_{\psi-\phi_{1} \phi_{2}} G^{\psi \phi_{3}} V_{\phi_{1} \phi_{2}-\psi}\left(G_{0}^{\phi \phi \phi} \hat{t}_{3}+1\right),
$$

where $\hat{t}_{3}(E)$ is defined by

$$
\hat{t}_{3}=V_{3} \frac{1}{1-G_{0}^{\phi \phi \phi} V_{3}} .
$$

Diagrammatic representation of $t_{3}$ is given as

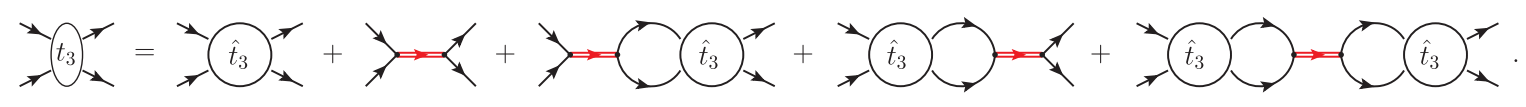

For notational simplicity, we rewrite $Z_{4}$ as follows:

$$
Z_{4}=z_{4} 1, \quad z_{4}=G_{0}^{\phi \phi \phi} V_{\psi-\phi_{1} \phi_{2}} \mathcal{G} V_{\phi_{1} \phi_{2}-\psi} G_{0}^{\phi \phi \phi},
$$

where we defined $\mathcal{G}$ by

$$
\mathcal{G}=G_{0}^{\psi \phi_{3}} t_{\psi \phi_{3}} \frac{1}{1-G_{0}^{\psi \phi_{3}} \Sigma_{0} t_{\psi \phi_{3}}} G_{0}^{\psi \phi_{3}}
$$

and $t_{\psi \phi_{3}}$ by

$$
t_{\psi \phi_{3}}=V_{\psi \phi_{3}}+V_{\psi \phi_{3}} \frac{1}{E-H_{0}^{Q}-V_{\psi \phi_{3}}} V_{\psi \phi_{3}}
$$

whose diagrammatic representation is

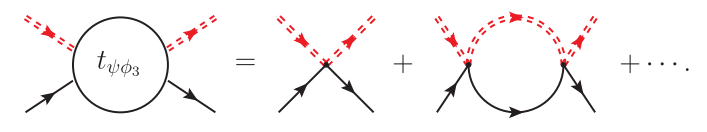

$z_{4} t_{3} z_{4}$, which appears in higher-order terms of the effective AGS equations, is written as

$$
\begin{aligned}
& z_{4} t_{3} z_{4}=G_{0}^{\phi \phi \phi} V_{\psi-\phi_{1} \phi_{2}} \mathcal{G}\left(\Sigma_{V}+\Sigma G^{\psi \phi_{3}} \Sigma\right) \mathcal{G} V_{\psi-\phi_{1} \phi_{2}} G_{0}^{\phi \phi \phi}
\end{aligned}
$$

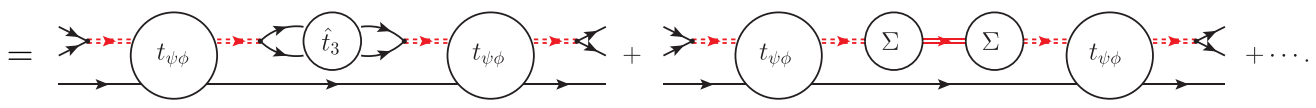

The corresponding $\Sigma_{0}$ for the first term in the above is actually included in the second term of $z_{4}$ as

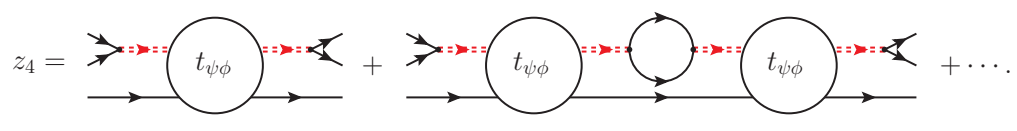

Combining the second term of the above equation with the first term of Eq. (A10), we obtain

$$
\begin{aligned}
& G_{0}^{\phi \phi \phi} V_{\psi-\phi_{1} \phi_{2}} G_{0}^{\psi \phi_{3}} t_{\psi \phi_{3}} G_{0}^{\psi \phi_{3}}\left(\Sigma+\Sigma G^{\psi \phi_{3}} \Sigma\right) G_{0}^{\psi \phi_{3}} t_{\psi \phi_{3}} G_{0}^{\psi \phi_{3}} V_{\phi_{1} \phi_{2}-\psi} G_{0}^{\phi \phi \phi}
\end{aligned}
$$

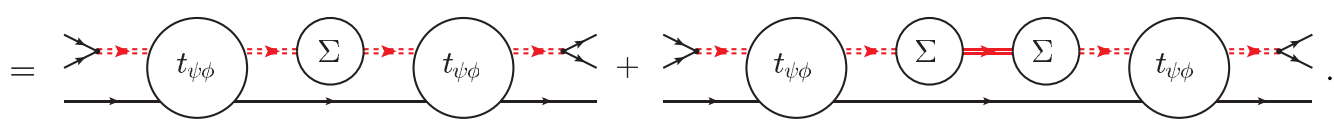


The corresponding counterterms are as follows:

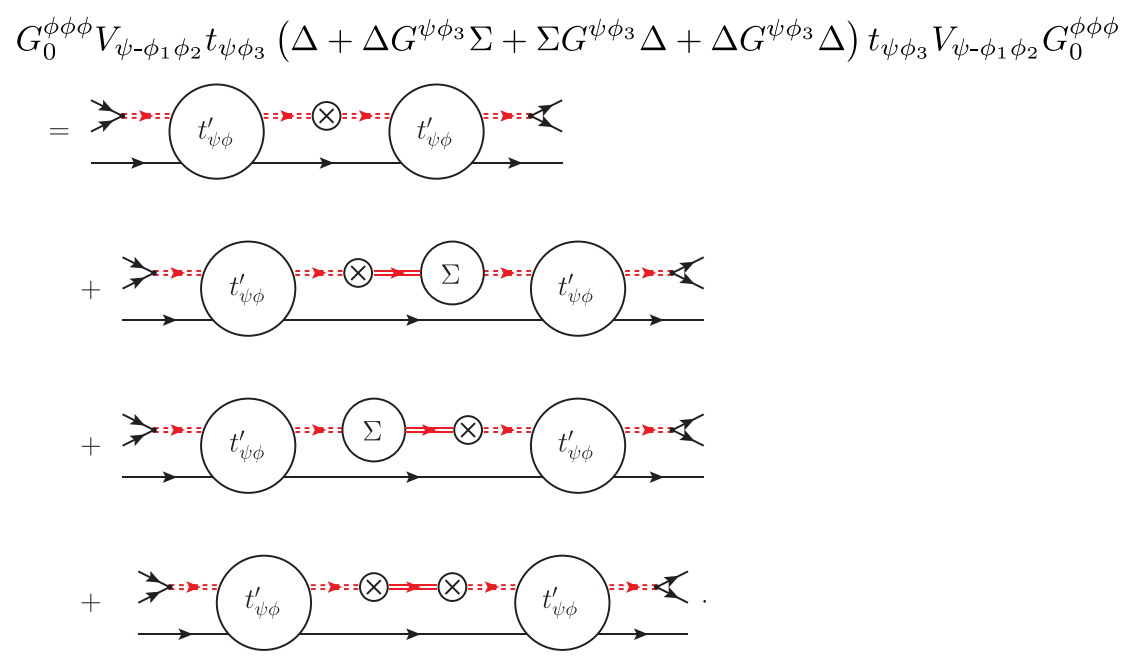

Another way the self-energies appear in higher-order terms of the effective AGS equations is in ones like $Z_{0} T Z_{4}$, which contains the matrix elements $G_{0}^{\phi \phi \phi} t_{3} z_{4}$ which are rewritten as

$$
\begin{aligned}
G_{0}^{\phi \phi \phi} t_{3} z_{4} & =G_{0}^{\phi \phi \phi}\left(\hat{t}_{3}+\left(1+\hat{t}_{3} G_{0}^{\phi \phi \phi}\right) V_{\psi-\phi_{1} \phi_{2}} G^{\psi \phi_{3}} V_{\phi_{1} \phi_{2}-\psi}\left(G_{0}^{\phi \phi \phi} \hat{t}_{3}+1\right)\right) G_{0}^{\phi \phi \phi} V_{\psi-\phi_{1} \phi_{2}} \mathcal{G} V_{\psi-\phi_{1} \phi_{2}} G_{0}^{\phi \phi \phi} \\
& =G_{0}^{\phi \phi \phi}\left(\hat{t}_{3} G_{0}^{\phi \phi \phi} V_{\psi-\phi_{1} \phi_{2}}+\left(1+\hat{t}_{3} G_{0}^{\phi \phi \phi}\right) V_{\psi-\phi_{1} \phi_{2}} G^{\psi \phi_{3}} \Sigma\right) \mathcal{G} V_{\psi-\phi_{1} \phi_{2}} G_{0}^{\phi \phi \phi},
\end{aligned}
$$

whose diagrammatic representation is given below:

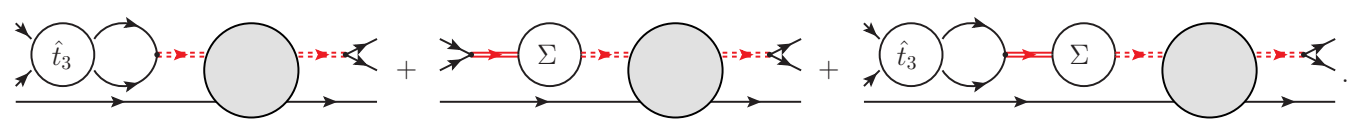

The corresponding counterterms are, therefore,

$$
G_{0}^{\phi \phi \phi}\left(1+\hat{t}_{3} G_{0}^{\phi \phi \phi}\right) V_{\psi-\phi_{1} \phi_{2}} G^{\psi \phi_{3}} \Delta \mathcal{G} V_{\psi-\phi_{1} \phi_{2}} G_{0}^{\phi \phi \phi}=
$$

Having seen what sort of self-energies appear in higher-order terms of the effective AGS equations, we find a way to put those counterterms into one of the driving terms $Z_{4}$ and define the modified one in the next section.

\section{Reorganization of the effective AGS equations and the modified kernel}

In the following, we perform the mass renormalization and reorganize scattering processes in the effective AGS equations so as to keep the structure of the equations the same while the counterterms in higher-order terms of the equations are included into the kernel of the equations.

The self-energies appearing in higher-order terms of the effective AGS equations are generated when one of the driving terms, $Z_{4}$, and one of the two-body $T$-matrices, $t_{3}$, are multiplied. We therefore sum up $t_{3}$ first so that the additional self-energy does not appear in higher-order terms as discussed in detail below.

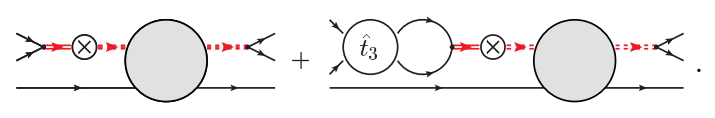

As we saw in Sec. II, the transition amplitude $X$ is reorganized as

$$
X=\frac{1}{1-Z T} Z=X_{3} \frac{1}{1-\bar{T}_{3} X_{3}} .
$$

Noting that $Z_{0} T_{3} Z_{0}=0$, we can simplify $X_{3}$ as

$$
X_{3}=Z_{0}+Z_{0} T_{3} Z_{0}+\left(1+Z_{0} T_{3}\right) W_{3}\left(T_{3} Z_{0}+1\right),
$$

where we defined $W_{3}$ by

$$
W_{3}=Z_{4} \frac{1}{1-T_{3} Z_{4}} .
$$

We note that the additional self-energy does not appear when $\bar{T}_{3}$ and $X_{3}$ are multiplied. $W_{3}$ is channel independent, $W_{3}=w_{3} 1$, where $w_{3}$ is defined by

$$
w_{3}=z_{4}+z_{4} t_{3} z_{4}+\cdots=z_{4} \frac{1}{1-t_{3} z_{4}} .
$$

$w_{3}$ can be expressed in some ways. Substituting an expression in Eq. (44), $w_{3}$ is written as 


$$
\begin{aligned}
w_{3} & =G_{0}^{\phi \phi \phi} V_{\psi-\phi_{1} \phi_{2}} \mathcal{G} \frac{1}{1-V_{\phi_{1} \phi_{2}-\psi} G_{0}^{\phi \phi \phi} t_{3} G_{0}^{\phi \phi \phi} V_{\psi-\phi_{1} \phi_{2}} \mathcal{G}} V_{\phi_{1} \phi_{2}-\psi} G_{0}^{\phi \phi \phi} \\
& =G_{0}^{\phi \phi \phi} V_{\psi-\phi_{1} \phi_{2}} \mathcal{G} \frac{1}{1-\left(\Sigma_{V}+\Sigma G^{\psi \phi} \phi_{3} \Sigma\right) \mathcal{G}} V_{\phi_{1} \phi_{2}-\psi} G_{0}^{\phi \phi \phi}
\end{aligned}
$$

Substituting the definition of $\mathcal{G}$ [Eq. (A7)], we obtain the following expression:

$$
\begin{aligned}
& w_{3}=G_{0}^{\phi \phi \phi} V_{\psi-\phi_{1} \phi_{2}} G_{0}^{\psi \phi_{3}} t_{\psi \phi_{3}} G_{0}^{\psi \phi_{3}} \frac{1}{1-\left(\Sigma+\Sigma G^{\psi \phi_{3}} \Sigma\right) G_{0}^{\psi \phi_{3}} t_{\psi \phi_{3}} G_{0}^{(2)}} V_{\phi_{1} \phi_{2}-\psi} G_{0}^{\phi \phi \phi}
\end{aligned}
$$

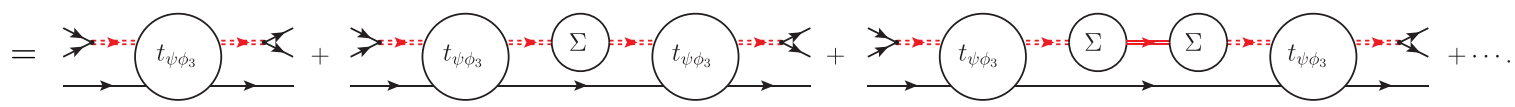

Noting that

$$
G_{0}^{\psi \phi_{3}}\left(\Sigma+\Sigma G^{\psi \phi_{3}} \Sigma\right) G_{0}^{\psi \phi_{3}}=G^{\psi \phi_{3}}-G_{0}^{\psi \phi_{3}}
$$

we obtain another expression:

$$
\begin{aligned}
& w_{3}=G_{0}^{\phi \phi \phi} V_{\psi-\phi_{1} \phi_{2}} G_{0}^{\psi \phi_{3}} t_{\psi \phi_{3}} \frac{1}{1-\left(G^{\psi \phi_{3}}-G_{0}^{\psi \phi_{3}}\right) t_{\psi \phi_{3}}} G_{0}^{\psi \phi_{3}} V_{\phi_{1} \phi_{2}-\psi} G_{0}^{\phi \phi \phi}
\end{aligned}
$$

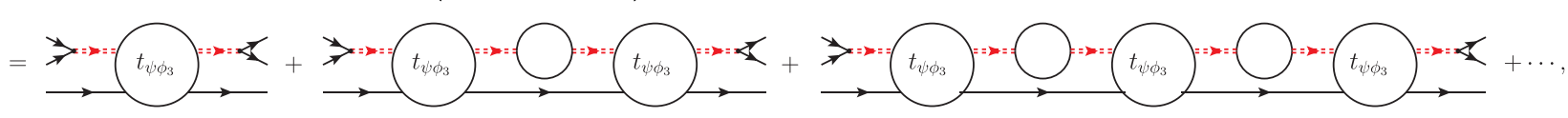

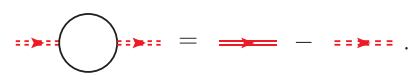

Substituting the definition of $t_{\psi \phi_{3}}$, we have the most intuitive expression of $w_{3}$ :

$$
\begin{aligned}
w_{3} & =G_{0}^{\phi \phi \phi} V_{\psi-\phi_{1} \phi_{2}} G_{0}^{\psi \phi_{3}} V_{\psi \phi_{3}} \frac{1}{1-G^{\psi \phi_{3}} V_{\psi \phi_{3}}} G_{0}^{\psi \phi_{3}} V_{\phi_{1} \phi_{2}-\psi} G_{0}^{\phi \phi \phi} \\
& =G_{0}^{\phi \phi \phi} V_{\psi-\phi_{1} \phi_{2}} G_{0}^{\psi \phi_{3}} t_{\psi \phi_{3}}^{\Sigma} G_{0}^{\psi \phi_{3}} V_{\phi_{1} \phi_{2}-\psi} G_{0}^{\phi \phi \phi}
\end{aligned}
$$

The intermediate factor $t_{\psi \phi_{3}}^{\Sigma}=V_{\psi \phi_{3}} /\left(1-G^{\psi \phi_{3}} V_{\psi \phi_{3}}\right)$ is represented in terms of the renormalized quantities as follows:

$$
\begin{aligned}
t_{\psi \phi_{3}}^{\Sigma} & =t_{\psi \phi_{3}}^{\prime}+t_{\psi \phi_{3}}^{\prime}\left(G^{\psi \phi_{3}}-G_{0}^{\prime \psi \phi_{3}}\right) t_{\psi \phi_{3}}^{\prime}+\cdots \\
& =t_{\psi \phi_{3}}^{\prime}+t_{\psi \phi_{3}}^{\prime} G_{0}^{\prime \psi \phi_{3}}\left(\Sigma+\Delta+(\Sigma+\Delta) G^{\psi \phi_{3}}(\Sigma+\Delta)\right) G_{0}^{\prime} \psi \phi_{3} t_{\psi \phi_{3}}^{\prime}+\cdots,
\end{aligned}
$$

where we introduced the renormalized free Green function

$$
G_{0}^{\prime \psi \phi_{3}}=\left(E-M^{\prime}-m_{3}-\frac{p_{3}^{2}}{2 M^{\prime}}-\frac{k_{3}^{2}}{2 m_{3}}\right)^{-1}
$$

and another two-body $T$-matrix,

$$
t_{\psi \phi_{3}}^{\prime}=V_{\psi \phi_{3}}+V_{\psi \phi_{3}} G_{0}^{\prime} \psi \phi_{3} V_{\psi \phi_{3}}+\cdots .
$$

Its Green function is the bare one instead of the dressed one. $w_{3}$ is therefore written in terms of the renormalized quantities as

$$
\begin{aligned}
w_{3} & =G_{0}^{\phi \phi \phi} V_{\psi-\phi_{1} \phi_{2}} G_{0}^{\psi \phi_{3}} t_{\psi \phi_{3}}^{\prime} \frac{1}{1-\left(G^{\psi \phi_{3}}-G_{0}^{\prime \psi \phi_{3}}\right) t_{\psi \phi_{3}}^{\prime}} G_{0}^{\psi \phi_{3}} V_{\phi_{1} \phi_{2}-\psi} G_{0}^{\phi \phi \phi} \\
& =G_{0}^{\phi \phi \phi} V_{\psi-\phi_{1} \phi_{2}} G_{0}^{\psi \phi_{3}} t_{\psi \phi_{3}}^{\prime} \frac{1}{1-G_{0}^{\prime \psi \phi_{3}}\left(\Sigma+\Delta+(\Sigma+\Delta) G^{\psi \phi_{3}}(\Sigma+\Delta)\right) G_{0}^{\prime \psi \phi_{3}} t_{\psi \phi_{3}}^{\prime}} G_{0}^{\psi \phi_{3}} V_{\phi_{1} \phi_{2}-\psi} G_{0}^{\phi \phi \phi}
\end{aligned}
$$




$$
\begin{aligned}
& =G_{0}^{\phi \phi \phi} V_{\psi-\phi_{1} \phi_{2}} \mathcal{G}^{\prime} \frac{1}{1-\left(\Sigma_{V}+\Sigma G_{\Sigma}^{\psi \phi_{3}} \Sigma\right) \mathcal{G}^{\prime}} V_{\phi_{1} \phi_{2}-\psi} G_{0}^{\phi \phi \phi} \\
& =G_{0}^{\phi \phi \phi} V_{\psi-\phi_{1} \phi_{2}} \mathcal{G}^{\prime} \frac{1}{1-V_{\phi_{1} \phi_{2}-\psi} G_{0}^{\phi \phi \phi} t_{3} G_{0}^{\phi \phi \phi} V_{\psi-\phi_{1} \phi_{2}} \mathcal{G}^{\prime}} V_{\phi_{1} \phi_{2}-\psi} G_{0}^{\phi \phi \phi},
\end{aligned}
$$

where we defined $\mathcal{G}^{\prime}$ by

$$
\begin{aligned}
\mathcal{G}^{\prime} & =G_{0}^{\prime \psi \phi_{3}}\left(t_{\psi \phi_{3}}^{\prime}+t_{\psi \phi_{3}}^{\prime} G_{0}^{\prime \psi \phi_{3}}\left(\Sigma_{0}+\Delta+\Delta G^{\psi \phi_{3}} \Sigma+\Sigma G^{\psi \phi_{3}} \Delta+\Delta G^{\psi \phi_{3}} \Delta\right) G_{0}^{\prime} \psi \phi_{3} t_{\psi \phi_{3}}^{\prime}+\cdots\right) G_{0}^{\prime \psi \phi_{3}} \\
& =G_{0}^{\prime \psi \phi_{3}}\left(t_{\psi \phi_{3}}^{\prime}+t_{\psi \phi_{3}}^{\prime}\left(G^{\psi \phi_{3}}-G_{0}^{\prime \psi \phi_{3}}-G_{0}^{\prime \psi \phi_{3}}\left(\Sigma_{V}+\Sigma G^{\psi \phi_{3}} \Sigma\right) G_{0}^{\prime \psi \phi_{3}}\right) t_{\psi \phi_{3}}^{\prime}+\cdots\right) G_{0}^{\prime \psi \phi_{3}} .
\end{aligned}
$$

Its diagrammatic expression is given as

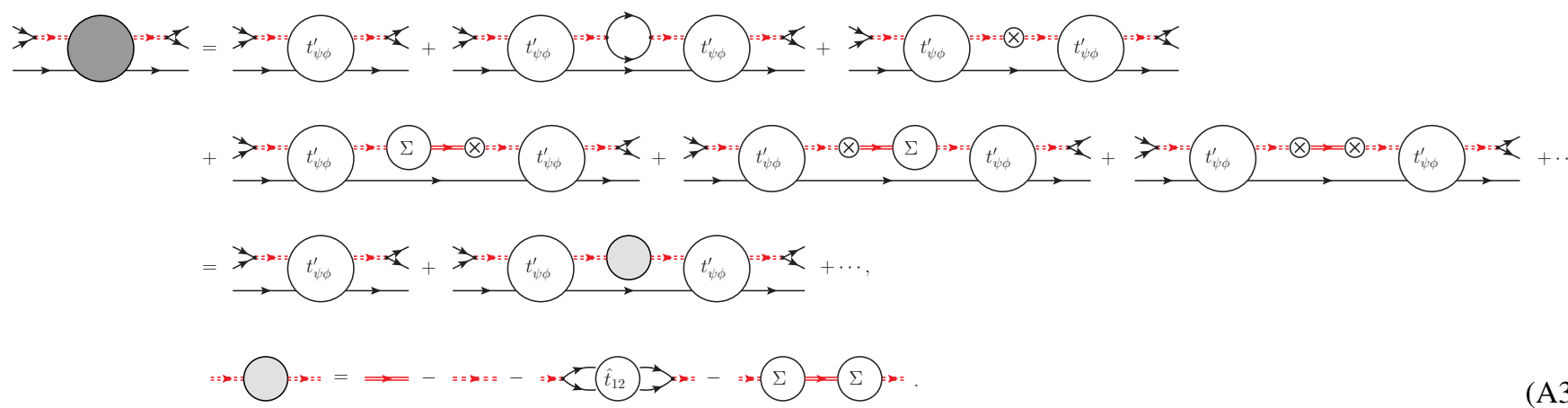

With $\mathcal{G}^{\prime}$ defined above, $w_{3}$ is written as

$$
w_{3}=G_{0}^{\phi \phi \phi} V_{\psi-\phi_{1} \phi_{2}} \mathcal{G}^{\prime} V_{\phi_{1} \phi_{2}-\psi} G_{0}^{\phi \phi \phi}+G_{0}^{\phi \phi \phi} V_{\psi-\phi_{1} \phi_{2}} \mathcal{G}^{\prime} V_{\phi_{1} \phi_{2}-\psi} G_{0}^{(3)} t_{3} G_{0}^{\phi \phi \phi} V_{\psi-\phi_{1} \phi_{2}} \mathcal{G}^{\prime} V_{\phi_{1} \phi_{2}-\psi} G_{0}^{\phi \phi \phi}+\cdots .
$$

We can see that the counterterms in higher-order terms of the effective AGS equations are now put into $\mathcal{G}^{\prime}$ while keeping the form of the effective AGS equations the same.

We have been focused on the intermediate part of the scattering processes. We next discuss the left- and right-most parts of the scattering processes which have the channel-dependent structure. Explicit expressions for the left- and right-most structures of the last term in $W_{3}$ are

$$
1+Z_{0} T_{3}=\left(\begin{array}{ccc}
1 & 0 & G_{0}^{\phi \phi \phi} t_{3} \\
0 & 1 & G_{0}^{\phi \phi \phi} t_{3} \\
0 & 0 & 1
\end{array}\right), \quad T_{3} Z_{0}+1=\left(\begin{array}{ccc}
1 & 0 & 0 \\
0 & 1 & 0 \\
G_{0}^{\phi \phi \phi} t_{3} & G_{0}^{\phi \phi \phi} t_{3} & 1
\end{array}\right) .
$$

The explicit matrix expression for $\left(1+Z_{0} T_{3}\right) W_{3}\left(T_{3} Z_{0}+1\right)$ is therefore

$$
\left(\begin{array}{ccc}
\left(1+G_{0}^{\phi \phi \phi} t_{3}\right) w_{3}\left(t_{3} G_{0}^{\phi \phi \phi}+1\right) & \left(1+G_{0}^{\phi \phi \phi} t_{3}\right) w_{3}\left(t_{3} G_{0}^{\phi \phi \phi}+1\right) & \left(1+G_{0}^{\phi \phi \phi} t_{3}\right) w_{3} \\
\left(1+G_{0}^{\phi \phi \phi} t_{3}\right) w_{3}\left(t_{3} G_{0}^{\phi \phi \phi}+1\right) & \left(1+G_{0}^{\phi \phi \phi} t_{3}\right) w_{3}\left(t_{3} G_{0}^{\phi \phi \phi}+1\right) & \left(1+G_{0}^{\phi \phi \phi} t_{3}\right) w_{3} \\
w_{3}\left(t_{3} G_{0}^{\phi \phi \phi}+1\right) & w_{3}\left(t_{3} G_{0}^{\phi \phi \phi}+1\right) & w_{3}
\end{array}\right)
$$

Noting that the left- and the right-most factors of $w_{3}$ are $G_{0}^{\phi \phi \phi} V_{\psi-\phi_{1} \phi_{2}}$ and $V_{\phi_{1} \phi_{2}-\psi} G_{0}^{(3)}$, we obtain

$$
\begin{aligned}
& \left(1+G_{0}^{\phi \phi \phi} t_{3}\right) G_{0}^{\phi \phi \phi} V_{\psi-\phi_{1} \phi_{2}}=G_{0}^{\phi \phi \phi}\left(1+\hat{t}_{3} G_{0}^{\phi \phi \phi}\right) V_{\psi-\phi_{1} \phi_{2}}\left(1+G^{\psi \phi_{3}} \Sigma\right), \\
& V_{\phi_{1} \phi_{2}-\psi} G_{0}^{\phi \phi \phi}\left(t_{3} G_{0}^{\phi \phi \phi}+1\right)=\left(\Sigma G^{\psi \phi_{3}}+1\right) V_{\phi_{1} \phi_{2}-\psi}\left(G_{0}^{\phi \phi \phi} \hat{t}_{3}+1\right) G_{0}^{\phi \phi \phi} .
\end{aligned}
$$

The $(1,1)$ component, for example, is then written as

$$
\begin{aligned}
& G_{0}^{\phi \phi \phi}\left(1+\hat{t}_{3} G_{0}^{\phi \phi \phi}\right) V_{\psi-\phi_{1} \phi_{2}}\left(1+G^{\psi \phi_{3}} \Sigma\right) G_{0}^{\psi \phi_{3}} t_{\psi \phi_{3}}^{\Sigma} G_{0}^{\psi \phi_{3}}\left(\Sigma G^{\psi \phi_{3}}+1\right) V_{\phi_{1} \phi_{2}-\psi}\left(G_{0}^{\phi \phi \phi} \hat{t}_{3}+1\right) G_{0}^{\phi \phi \phi} \\
& =G_{0}^{\phi \phi \phi}\left(1+\hat{t}_{3} G_{0}^{\phi \phi \phi}\right) V_{\psi-\phi_{1} \phi_{2}} G^{\psi \phi_{3}} t_{\psi \phi_{3}}^{\Sigma} G^{\psi \phi_{3}} V_{\phi_{1} \phi_{2}-\psi}\left(G_{0}^{\phi \phi \phi} \hat{t}_{3}+1\right) G_{0}^{\phi \phi \phi} .
\end{aligned}
$$

Similarly, the $(1,3)$ component is written as

$$
G_{0}^{\phi \phi \phi}\left(1+\hat{t}_{3} G_{0}^{\phi \phi \phi}\right) V_{\psi-\phi_{1} \phi_{2}} G^{\psi \phi_{3}} t_{\psi \phi_{3}}^{\Sigma} G_{0}^{\psi \phi_{3}} V_{\phi_{1} \phi_{2}-\psi} G_{0}^{\phi \phi \phi},
$$

and the $(3,1)$ component as

$$
G_{0}^{\phi \phi \phi} V_{\psi-\phi_{1} \phi_{2}} G_{0} t_{\psi \phi_{3}}^{\Sigma} G^{\psi \phi_{3}} V_{\phi_{1} \phi_{2}-\psi}\left(G_{0}^{\phi \phi \phi} \hat{t}_{3}+1\right) G_{0}^{\phi \phi \phi} .
$$


The diagrammatic representation of each matrix element is given as
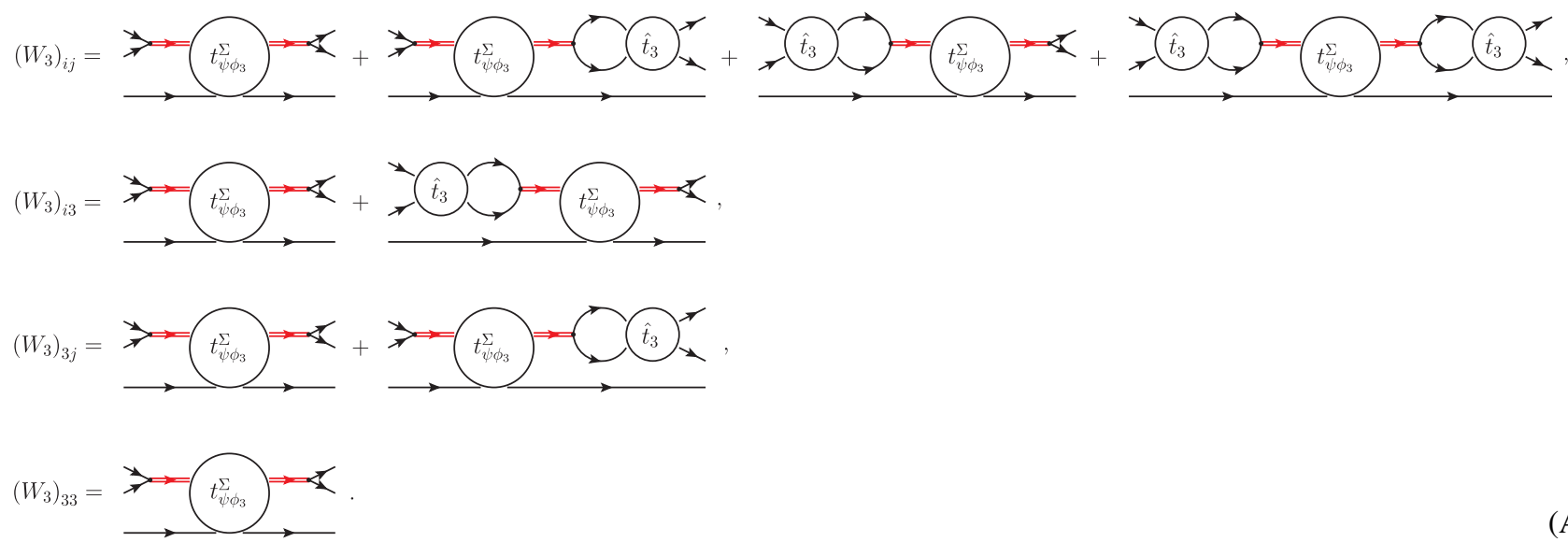

We now rewrite them in terms of the renormalized quantities as we did in the previous paragraph. For example, the $(1,3)$ component is written as

$$
\begin{aligned}
& G_{0}^{\phi \phi \phi}\left(1+\hat{t}_{3} G_{0}^{\phi \phi \phi}\right) V_{\psi-\phi_{1} \phi_{2}} G^{\psi \phi_{3}} t_{\psi \phi_{3}}^{\Sigma} G_{0}^{\psi \phi_{3}} V_{\phi_{1} \phi_{2}-\psi} G_{0}^{\phi \phi \phi} \\
& \quad=G_{0}^{\phi \phi \phi}\left(1+\hat{t}_{3} G_{0}^{\phi \phi \phi}\right) V_{\psi-\phi_{1} \phi_{2}}\left(1+G^{\psi \phi_{3}}(\Sigma+\Delta)\right) G_{0}^{\psi}{ }^{\psi \phi_{3}} t_{\psi \phi_{3}}^{\Sigma} G_{0}^{\psi \phi_{3}} V_{\phi_{1} \phi_{2}-\psi} .
\end{aligned}
$$

We include the counterterm by modifying the left-most part of the kernel as

$$
G_{0}^{\phi \phi \phi}\left(1+\hat{t}_{3} G_{0}^{\phi \phi \phi}\right) V_{\psi-\phi_{1} \phi_{2}}\left(1+G^{\psi \phi_{3}} \Delta\right) \mathcal{G}^{\prime} V_{\phi_{1} \phi_{2}-\psi} G_{0}^{\phi \phi \phi} .
$$

For the $(3,1)$ component, we modify the kernel as

$$
G_{0}^{\phi \phi \phi} V_{\psi-\phi_{1} \phi_{2}} \mathcal{G}^{\prime}\left(\Delta G^{\psi \phi_{3}}+1\right) V_{\phi_{1} \phi_{2}-\psi}\left(G_{0}^{\phi \phi \phi} \hat{t}_{3}+1\right) G_{0}^{\phi \phi \phi},
$$

and for the $(1,1)$ component as

$$
G_{0}^{\phi \phi \phi}\left(1+\hat{t}_{3} G_{0}^{\phi \phi \phi}\right) V_{\psi-\phi_{1} \phi_{2}}\left(1+G^{\psi \phi_{3}} \Delta\right) \mathcal{G}^{\prime}\left(\Delta G^{\psi \phi_{3}}+1\right) V_{\phi_{1} \phi_{2}-\psi}\left(G_{0}^{\phi \phi \phi} \hat{t}_{3}+1\right) G_{0}^{\phi \phi \phi} .
$$

Each component of the modified driving term is as follows:

$$
\begin{aligned}
& \left(Z_{4}^{\prime}\right)_{i j}=G_{0}^{\phi \phi \phi}\left(1+\hat{t}_{3} G_{0}^{\phi \phi \phi}\right) V_{\psi-\phi_{1} \phi_{2}}\left(1+G^{\psi \phi_{3}} \Delta\right) \mathcal{G}^{\prime}\left(\Delta G^{\psi \phi_{3}}+1\right) V_{\phi_{1} \phi_{2}-\psi}\left(G_{0}^{\phi \phi \phi} \hat{t}_{3}+1\right) G_{0}^{\phi \phi \phi} \quad(i, j=1,2), \\
& \left(Z_{4}^{\prime}\right)_{i 3}=G_{0}^{\phi \phi \phi}\left(1+\hat{t}_{3} G_{0}^{\phi \phi \phi}\right) V_{\psi-\phi_{1} \phi_{2}}\left(1+G^{\psi \phi_{3}} \Delta\right) \mathcal{G}^{\prime} V_{\phi_{1} \phi_{2}-\psi} G_{0}^{\phi \phi \phi} \quad(i=1,2), \\
& \left(Z_{4}^{\prime}\right)_{3 j}=G_{0}^{\phi \phi \phi} V_{\psi-\phi_{1} \phi_{2}} \mathcal{G}^{\prime}\left(\Delta G^{\psi \phi_{3}}+1\right) V_{\phi_{1} \phi_{2}-\psi}\left(G_{0}^{\phi \phi \phi} \hat{t}_{3}+1\right) G_{0}^{\phi \phi \phi} \quad(j=1,2), \\
& \left(Z_{4}^{\prime}\right)_{33}=G_{0}^{\phi \phi \phi} V_{\psi-\phi_{1} \phi_{2}} \mathcal{G}^{\prime} V_{\phi_{1} \phi_{2}-\psi} G_{0}^{\phi \phi \phi} .
\end{aligned}
$$

The diagrammatic representation of each component is given in Fig. 8.
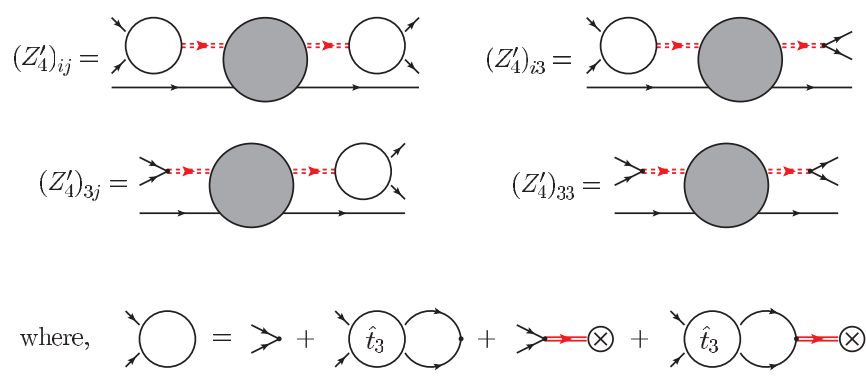

FIG. 8. A diagrammatic representation of the modified kernel.

\section{APPENDIX B: JACOBI MOMENTA AND EXPLICIT EXPRESSIONS FOR THE MATRIX ELEMENTS IN THE EFFECTIVE AGS EQUATIONS}

\section{Jacobi momenta}

In a three-body system, it is convenient to introduce the Jacobi momenta defined by

$$
\begin{aligned}
\mathbf{P} & =\mathbf{k}_{1}+\mathbf{k}_{2}+\mathbf{k}_{3}, \\
\mathbf{q}_{i} & =\frac{\left(m_{j}+m_{k}\right) \mathbf{k}_{i}-m_{i}\left(\mathbf{k}_{j}+\mathbf{k}_{k}\right)}{m_{i}+m_{j}+m_{k}}, \\
\mathbf{p}_{i} & =\frac{m_{k} \mathbf{k}_{j}-m_{j} \mathbf{k}_{k}}{m_{j}+m_{k}},
\end{aligned}
$$

where $i, j, k$ are cyclic permutations of $1,2,3$ and $\mathbf{k}_{i}$ is a momentum of $\phi_{i}$ in Cartesian coordinates. In the following, we 
consider the problem in the three-body center-of-mass frame; that is, we set

$$
\mathbf{P}=\mathbf{0}
$$

The other two Jacobi momenta then become

$$
\begin{aligned}
\mathbf{q}_{i} & =\mathbf{k}_{i}, \\
\mathbf{p}_{i} & =\frac{m_{k} \mathbf{k}_{j}-m_{j} \mathbf{k}_{k}}{m_{j}+m_{k}} .
\end{aligned}
$$

We also introduce reduced masses as follows:

$$
\begin{aligned}
& M_{i}^{-1}=\left(m_{j}+m_{k}\right)^{-1}+m_{i}^{-1}, \\
& \mu_{\phi_{j} \phi_{k}}^{-1}=m_{j}^{-1}+m_{k}^{-1} .
\end{aligned}
$$

With this notation we introduced, the kinetic energy in the three-body channel in the center-of-mass frame is written as

$$
\begin{aligned}
& \sum_{i=1}^{3}\left(m_{i}+\frac{k_{i}^{2}}{2 m_{i}}\right) \\
& \quad=\sum_{i=1}^{3} m_{i}+\frac{P^{2}}{2 \sum_{i=1}^{3} m_{i}}+\frac{q_{i}^{2}}{2 M_{i}}+\frac{p_{i}^{2}}{2 \mu_{\phi_{j} \phi_{k}}} \\
& \quad=\sum_{i=1}^{3} m_{i}+\frac{q_{i}^{2}}{2 M_{i}}+\frac{p_{i}^{2}}{2 \mu_{\phi_{j} \phi_{k}}} .
\end{aligned}
$$

We denote the center of mass and the relative momentum in the two-body channel as

$$
\begin{aligned}
& \mathcal{P}=\mathbf{K}_{3}+\mathbf{k}_{3}, \\
& \mathbf{p}=\frac{m_{3} \mathbf{K}_{3}-M \mathbf{k}_{3}}{M+m_{3}} .
\end{aligned}
$$

We denote the reduced mass of $\psi \phi_{3}$ as $\mu_{\psi \phi_{3}}$ :

$$
\left(\mu_{\psi \phi_{3}}\right)^{-1}=M^{-1}+m_{3}^{-1}
$$

where we placed a superscript on the mass of $\psi$ since its mass shifts from the bare one by the coupling to the $\phi_{1} \phi_{2}$ two-body state. The kinetic energy in the two-body channel in the centerof-mass frame is therefore written as

$$
\begin{aligned}
M & +\frac{K_{3}^{2}}{2 M}+m_{3}+\frac{k_{3}^{2}}{2 m_{3}} \\
& =M+m_{3}+\frac{\mathcal{P}^{2}}{2\left(M+m_{3}\right)}+\frac{p^{2}}{2 \mu_{\psi \phi_{3}}} \\
& =M+m_{3}+\frac{p^{2}}{2 \mu_{\psi \phi_{3}}} .
\end{aligned}
$$

In Sec. III, we perform a numerical calculation in the case where the two-body and three-body thresholds are degenerate,

$$
M^{\prime}=m_{1}+m_{2},
$$

where we introduced the physical mass of $\psi, M^{\prime}$. We briefly discuss how each kinetic quantities are related in that case. If two thresholds are degenerate, we have

$$
\mathbf{K}_{3}=\mathbf{k}_{1}+\mathbf{k}_{2} \text {, }
$$

which leads to

$$
\mathbf{P}=\mathbf{k}_{1}+\mathbf{k}_{2}+\mathbf{k}_{3}=\mathbf{K}_{3}+\mathbf{k}_{3}=\mathcal{P} .
$$

That is, the three-body center-of-mass frame is also the twobody center-of-mass frame. The relative momentum between the pair $\phi_{1} \phi_{2}$ and $\phi_{3}$ is then

$$
\begin{aligned}
\mathbf{q}_{3} & =\frac{\left(m_{1}+m_{2}\right) \mathbf{k}_{3}-m_{3}\left(\mathbf{k}_{1}+\mathbf{k}_{2}\right)}{m_{1}+m_{2}+m_{3}} \\
& =\frac{M^{\prime} \mathbf{k}_{3}-m_{3} \mathbf{K}_{3}}{M^{\prime}+m_{3}}=-\mathbf{p} .
\end{aligned}
$$

In a word, the relative momentum between $\phi_{3}$ and $\phi_{1} \phi_{2}$ is equal (up to sign) to the relative momentum between $\psi$ and $\phi_{3}$.

\section{Elementary, effective interactions, $T$ matrices, and kernels}

In this section, we present explicit expressions for the elementary and the effective interactions and its matrix elements. Matrix elements of $Z_{0}(E)$ and (unmodified) $Z_{4}(E)$ are also presented.

Elementary interactions between $\phi_{j} \phi_{k}$ are of the form

$$
V_{\phi_{j} \phi_{k}}=\int_{0}^{\infty} q_{i}^{2} d q_{i}\left|q_{i} g\right\rangle \lambda_{\phi_{j} \phi_{k}}\left\langle q_{i} g\right|,
$$

and those coupling $\psi$ and $\phi_{1} \phi_{2}$ are

$$
V_{\psi-\phi_{1} \phi_{2}}=\int_{0}^{\infty} q_{3}^{2} d q_{3} \Gamma\left|q_{3}\right\rangle\left\langle q_{3} g\right|,
$$

and an interaction between $\psi \phi_{3}$ is

$$
V_{\psi \phi_{3}}=|g\rangle \lambda_{\psi \phi_{3}}\langle g|,
$$

where $|g\rangle$ is the Yamaguchi-type form factor. The effective interactions are therefore

$$
\begin{aligned}
U_{i}(E)= & V_{\phi_{j} \phi_{k}}=\int_{0}^{\infty} q_{i}^{2} d q_{i}\left|q_{i} g\right\rangle \lambda_{\phi_{j} \phi_{k}}\left\langle q_{i} g\right| \quad(i=1,2), \\
U_{3}(E)= & V_{\phi_{1} \phi_{2}}+V_{\psi-\phi_{1} \phi_{2}} G_{0}^{\psi \phi_{3}}(E) V_{\phi_{1} \phi_{2}-\psi} \\
= & \int_{0}^{\infty} q_{3}^{2} d q_{3}\left|q_{3} g\right\rangle\left(\lambda_{\phi_{1} \phi_{2}}+\Gamma G_{0}^{\psi \phi_{3}}\left(E q_{3}\right) \Gamma\right)\left\langle q_{3} g\right|, \\
U_{4}(E)= & V_{\psi-\phi_{1} \phi_{2}} G_{0}^{\psi \phi_{3}}(E) t_{\psi \phi_{3}}(E) G_{0}^{\psi \phi_{3}}(E) V_{\phi_{1} \phi_{2}-\psi} \\
= & \int_{0}^{\infty} q_{3}^{2} d q_{3} q_{3}^{\prime 2} d q_{3}^{\prime} \Gamma\left|q_{3} g\right\rangle G_{0}^{\psi \phi_{3}}\left(E q_{3}\right)\left\langle q_{3}\left|t_{\psi \phi_{3}}(E)\right| q_{3}^{\prime}\right\rangle \\
& \times G_{0}^{\psi \phi_{3}}\left(E q_{3}^{\prime}\right)\left\langle q_{3}^{\prime} g\right| \Gamma .
\end{aligned}
$$

As we saw in the previous Appendix, when it comes to considering the modified kernel in the presence of the elementary interactions between $\phi_{1} \phi_{2}$, it is convenient to express the two-body $T$-matrix of $\phi_{1} \phi_{2}$ as follows:

$$
\begin{aligned}
t_{3}(E)= & \hat{t}_{3}(E)+\left(1+\hat{t}_{3}(E) G_{0}^{\phi \phi \phi}(E)\right) \\
& \times V_{\psi-\phi_{1} \phi_{2}} G^{\psi \phi_{3}}(E) V_{\phi_{1} \phi_{2}-\psi}\left(G_{0}^{\phi \phi \phi}(E) \hat{t}_{3}(E)+1\right),
\end{aligned}
$$


which becomes

$$
\begin{aligned}
t_{3}(E)= & \int q_{3}^{2} d q_{3}\left|q_{3} g\right\rangle\left(\hat{\tau}_{3}\left(E q_{3}\right)\right. \\
& +\left(1+\hat{\tau}_{3}\left(E q_{3}\right) \mathcal{L}^{(3)}\left(E q_{3}\right)\right) \Gamma G^{\psi \phi_{3}}\left(E q_{3}\right) \\
& \left.\times \Gamma\left(\mathcal{L}^{(3)}\left(E q_{3}\right) \hat{\tau}_{3}\left(E q_{3}\right)+1\right)\right)\left\langle q_{3} g\right| .
\end{aligned}
$$

We defined a one-loop integral in the three-body channel,

$$
\mathcal{L}^{(3)}\left(E q_{3}\right)=\int p_{3}^{2} d p_{3} g^{2}\left(p_{3}\right) G_{0}^{\phi \phi \phi}\left(E q_{3} p_{3}\right),
$$

where $|g\rangle$ is the Yamaguchi-type form factor

$$
\langle p \mid g\rangle=g(p)=\frac{\Lambda^{2}}{p^{2}+\Lambda^{2}},
$$

and we introduced the two-body Green function,

$$
G_{0}^{\psi \phi_{3}}\left(E q_{3}\right)=\left(E-M^{\prime}-m_{3}-\frac{q_{3}^{2}}{2 \mu_{\psi \phi_{3}}}\right)^{-1} .
$$

Since $V_{\psi \phi_{3}}$ is separable, the $T$-matrix is expressed as follows:

$$
\begin{aligned}
t_{\psi \phi_{3}}(E) & =|g\rangle \tau_{\psi \phi_{3}}\langle g|, \\
\left(\tau_{\psi \phi_{3}}(E)\right)^{-1} & =\left(\lambda_{\psi \phi_{3}}\right)^{-1}-\frac{\pi}{2} \frac{\mu_{\psi \phi_{3}} \Lambda^{3}}{(k+i \Lambda)^{2}},
\end{aligned}
$$

where $k$ is the relative momentum between $\psi$ and $\phi_{3}$ defined by

$$
E=M^{\prime}+m_{3}+\frac{k^{2}}{2 \mu_{\psi \phi_{3}}} .
$$

It is convenient to define a dimensionless coupling constant, which we denote as $f_{\psi \phi_{3}}$ :

$$
f_{\psi \phi_{3}}=\lambda_{\psi \phi_{3}} \frac{\pi}{2} \mu_{\psi \phi_{3}} \Lambda
$$

$t_{\psi \phi_{3}}(E)$ has a bound-state pole for $f_{\psi \phi_{3}} \leqslant-1$ and a virtualstate pole for $f_{\psi \phi_{3}} \geqslant-1$. The effective three-body interaction $U_{4}(E)$ is then written as

$$
\begin{aligned}
U_{4}(E)= & \int_{0}^{\infty} q_{3}^{2} d q_{3} q_{3}^{\prime 2} d q_{3}^{\prime} \Gamma\left|q_{3} g\right\rangle G_{0}^{\psi \phi_{3}}\left(E q_{3}\right) g\left(q_{3}\right) \tau_{\psi \phi_{3}}(E) g \\
& \times\left(q_{3}^{\prime}\right) G_{0}^{\psi \phi_{3}}\left(E q_{3}^{\prime}\right)\left\langle q_{3}^{\prime} g\right| \Gamma .
\end{aligned}
$$

Since we assume separable interactions for two-body interactions among $\phi_{1} \phi_{2} \phi_{3}$, the two-body $T$-matrices are expressed as follows:

$$
t_{i}(E)=\int_{0}^{\infty} q_{i}^{2} d q_{i}\left|q_{i} g\right\rangle \tau_{i}\left(E q_{i}\right)\left\langle q_{i} g\right|,
$$

where

$$
\begin{aligned}
\left(\tau_{i}\left(E q_{i}\right)\right)^{-1} & =U_{i}^{-1}\left(E q_{i}\right)-\int_{0}^{\infty} p_{i}^{2} d p_{i} g^{2}\left(p_{i}\right) G_{0}^{\phi \phi \phi}\left(E q_{i} p_{i}\right) \\
& =U_{i}^{-1}\left(E q_{i}\right)-\frac{\pi}{2} \frac{\mu_{\phi_{j} \phi_{k}} \Lambda^{3}}{\left(k_{i}\left(E q_{i}\right)+i \Lambda\right)^{2}},
\end{aligned}
$$

and

$$
E=m_{i}+m_{j}+m_{k}+\frac{k_{i}^{2}\left(E q_{i}\right)}{2 \mu_{\phi_{j} \phi_{k}}}+\frac{q_{i}^{2}}{2 M_{i}} .
$$

$k_{i}$ is clearly the relative momentum between $\phi_{j} \phi_{k}$. We also present dimensionless coupling constants in the three-body channels as follows:

$$
f_{\phi_{j} \phi_{k}}=\lambda_{\phi_{j} \phi_{k}} \frac{\pi}{2} \mu_{\phi_{j} \phi_{k}} \Lambda .
$$

The three-body $T$-matrix is written as follows:

$$
\begin{aligned}
t_{4}(E)= & U_{4}(E)+U_{4}(E) G_{0}^{\phi \phi \phi}(E) U_{4}(E)+\cdots \\
= & \int q_{3}^{2} d q_{3} q_{3}^{\prime 2} d q_{3}^{\prime} \Gamma\left|q_{3} g\right\rangle G_{0}^{\psi \phi_{3}}\left(E q_{3}\right) g\left(q_{3}\right) \tau_{\psi \phi_{3}} \\
& \times \frac{1}{1-\left\langle g\left|G_{0}^{\psi \phi_{3}}(E) \Sigma_{0}(E) G_{0}^{\psi \phi_{3}}(E)\right| g\right\rangle \tau_{\psi \phi_{3}}(E)} \\
& \times g\left(q_{3}^{\prime}\right) G_{0}^{\psi \phi_{3}}\left(E q_{3}^{\prime}\right)\left\langle q_{3}^{\prime} g\right| \Gamma .
\end{aligned}
$$

The three-body $T$-matrix is written as follows:

$$
\begin{aligned}
t_{4}(E)= & U_{4}(E)+U_{4}(E) G_{0}^{\phi \phi \phi}(E) U_{4}(E)+\cdots \\
= & \int q_{3}^{2} d q_{3} q_{3}^{\prime 2} d q_{3}^{\prime} \Gamma\left|q_{3} g\right\rangle G_{0}^{\psi \phi_{3}}\left(E q_{3}\right) g\left(q_{3}\right) \tau_{\psi \phi_{3}} \\
& \times \frac{1}{1-\mathcal{V}(E) \tau_{\psi \phi_{3}}(E)} g\left(q_{3}^{\prime}\right) G_{0}^{\psi \phi_{3}}\left(E q_{3}^{\prime}\right)\left\langle q_{3}^{\prime} g\right| \Gamma,
\end{aligned}
$$

where we defined

$$
\mathcal{V}(E)=\left\langle g\left|G_{0}^{\psi \phi_{3}}(E) \Sigma_{0}(E) G_{0}^{\psi \phi_{3}}(E)\right| g\right\rangle .
$$

\section{The unmodified and the modified kernels}

The unmodified kernels of the AGS equations are therefore

$$
\begin{aligned}
Z_{0}(E) & =G_{0}^{\phi \phi \phi}(E) \bar{\delta}, \\
Z_{4}(E) & =G_{0}^{\phi \phi \phi}(E) t_{4}(E) \mathbf{1} G_{0}^{\phi \phi \phi}(E), \\
K(E) & =\left(Z_{0}(E)+Z_{4}(E)\right) T(E) .
\end{aligned}
$$

The modified $Z_{4}(E)$ are obtained as follows. First, we replace $\mathcal{V}(E)$ with

$$
\begin{aligned}
& \langle g| G_{0}^{\psi \phi_{3}}(E)\left(\Sigma(E)+\Sigma(E) G^{\psi \phi_{3}}(E) \Delta+\Delta G^{\psi \phi_{3}}(E) \Sigma(E)\right. \\
& \left.\quad+\Delta G^{\psi \phi_{3}}(E) \Delta\right) G_{0}^{\psi \phi_{3}}(E)|g\rangle,
\end{aligned}
$$

which can also be written as follows:

$$
\begin{aligned}
& \langle g| G^{\psi \phi_{3}}(E)-G_{0}^{\psi \phi_{3}}(E)-G_{0}^{\psi \phi_{3}}(E) \Sigma(E) G^{\psi \phi_{3}}(E) \\
& \quad \times \Sigma(E) G_{0}^{\psi \phi_{3}}(E)|g\rangle .
\end{aligned}
$$

For notational simplicity, we denote the above quantity as $\mathcal{V}(E)$. Second, we also replace the left-most structure with the following,

$$
\begin{aligned}
V_{\psi-\phi_{1} \phi_{2}} \Rightarrow & V_{\psi-\phi_{1} \phi_{2}}+\bar{\delta} I_{3}\left(1+\hat{t}_{3}(E) G_{0}^{\phi \phi \phi}(E)\right) \\
& \times V_{\psi-\phi_{1} \phi_{2}} G^{\psi \phi_{3}}(E) \Delta \\
= & V^{(32)^{\prime}}
\end{aligned}
$$

and the right-most structure with the corresponding one,

$$
\begin{aligned}
V_{\phi_{1} \phi_{2}-\psi} & \Rightarrow \Delta G^{\psi \phi_{3}}(E) V^{(23)}\left(G_{0}^{\phi \phi \phi}(E) \hat{t}_{3}(E)+1\right) I_{3} \bar{\delta}+V_{\psi-\phi_{1} \phi_{2}} \\
& =V^{(23)^{\prime}} .
\end{aligned}
$$


An explicit expression for the modified driving term $Z_{4}^{\prime}(E)$ is therefore obtained as follows:

$$
\begin{aligned}
Z_{4}^{\prime}(E)= & \int q_{3}^{2} d q_{3} q_{3}^{\prime 2} d q_{3}^{\prime} G_{0}^{\phi \phi \phi}(E) V^{(32)^{\prime}} G_{0}^{\psi \phi_{3}}(E)|g\rangle \tau_{\psi \phi_{3}}(E) \\
& \times \frac{1}{1-\mathcal{V}(E)}\langle g| \mathbf{1} G_{0}^{\psi \phi_{3}}(E) V^{(23)^{\prime}} G_{0}^{\phi \phi \phi}(E) .
\end{aligned}
$$

\section{Matrix elements of the driving terms}

In this section, we present an explicit expression of the matrix elements of the kernel. $Z_{0}\left(E q_{i} q_{j}\right)$ is represented as follows:

$$
\begin{aligned}
Z_{0}\left(E q_{i} q_{j}\right) & =\left\langle q_{j} g\left|\bar{\delta}_{i j} G_{0}^{\phi \phi \phi}(E)\right| q_{j} g\right\rangle \\
& =\frac{\bar{\delta}_{i j}}{2} \int_{-1}^{1} d x \frac{g\left(p_{i}\right) g\left(p_{j}\right)}{E-\frac{q_{i}^{2}}{2 m_{i}}-\frac{q_{j}^{2}}{2 m_{j}}-\frac{\left(\mathbf{q}_{i}+\mathbf{q}_{j}\right)^{2}}{2 m_{k}}},
\end{aligned}
$$

where $x$ and $\bar{\delta}_{i j}$ are defined by

$$
x=\hat{\mathbf{q}}_{\mathbf{i}} \cdot \hat{\mathbf{q}}_{\mathbf{j}}, \quad \bar{\delta}_{i j}=1-\delta_{i j} .
$$

$p_{i}$ and $p_{j}$ are the absolute values of relative momenta between $\phi_{j} \phi_{k}$ and $\phi_{k} \phi_{i}$, respectively, whose explicit expressions are

$$
p_{i}=\left|-\frac{m_{j}}{m_{j}+m_{k}} \mathbf{q}_{\mathbf{i}}-\mathbf{q}_{\mathbf{j}}\right|, \quad p_{j}=\left|\mathbf{q}_{\mathbf{i}}+\frac{m_{k}}{m_{k}+m_{i}} \mathbf{q}_{\mathbf{j}}\right| .
$$

Matrix elements of the unmodified $Z_{4}\left(E q_{3} q_{3}^{\prime}\right)$ are given as

$$
\begin{aligned}
& Z_{4}\left(E q_{3} q_{3}^{\prime}\right) \\
& \quad=\left\langle q_{3} g\left|G_{0}^{\phi \phi \phi}(E) t_{4}(E) G_{0}^{\phi \phi \phi}(E)\right| q_{3}^{\prime} g\right\rangle
\end{aligned}
$$

$$
\begin{aligned}
= & \int p_{3}^{2} d p_{3} g\left(p_{3}\right) G_{0}^{\phi \phi \phi}\left(E q_{3} p_{3}\right) \Gamma g\left(p_{3}\right) G_{0}^{\psi \phi_{3}}\left(E q_{3}\right) g \\
& \times\left(q_{3}\right) \tau_{\psi \phi_{3}}(E) \frac{1}{1-\mathcal{V}(E) \tau_{\psi \phi_{3}}(E)} g\left(q_{3}^{\prime}\right) G_{0}^{\psi \phi_{3}}\left(E q_{3}^{\prime}\right) \\
& \times \int p_{3}^{\prime 2} d p_{3}^{\prime} g\left(p_{3}^{\prime}\right) \Gamma G_{0}^{\phi \phi \phi}\left(E q_{3} p_{3}\right) g\left(p_{3}^{\prime}\right) .
\end{aligned}
$$

Other matrix elements of the unmodified $Z_{4}(E)$ are obtained in a straightforward way.

As we saw in the previous section, we need to perform the reorganization of the AGS equations and one of the kernel $Z_{4}(E)$ needs to be replaced by the modified one. $\mathcal{V}(E)$ is modified as

$$
\begin{aligned}
\mathcal{V}(E)= & \int q_{3}^{2} d q_{3}\left(G^{\psi \phi_{3}}\left(E q_{3}\right)-G_{0}^{\psi \phi_{3}}\left(E q_{3}\right)-G_{0}^{\psi \phi_{3}}\left(E q_{3}\right)\right. \\
& \left.\times\left(\Sigma_{V}(E)+\Sigma(E) G_{\Sigma}(E) \Sigma(E)\right) G_{0}^{\psi \phi_{3}}\left(E q_{3}\right)\right) .
\end{aligned}
$$

For matrix elements $Z_{4}\left(E q_{i} q_{j}\right)(i=1,2)$, the left-most structure of $Z_{4}(E)$ is modified as

$$
\begin{aligned}
& \int_{0}^{\infty} p_{3}^{2} d p_{3} g\left(p_{3}\right) G_{0}^{\phi \phi \phi}\left(E q_{3} p_{3}\right)\left(\Gamma g\left(p_{3}\right)\right. \\
& \left.+\left(1+\hat{\tau}_{3}(E) G_{0}^{\phi \phi \phi}\left(E q_{3} p_{3}\right)\right) \Gamma g\left(p_{3}\right) G^{\psi \phi_{3}}\left(E q_{3}\right) \Delta\right) .
\end{aligned}
$$

Similarly, for matrix elements $Z_{4}\left(E q_{i} q_{j}\right)(j=1,2)$ the rightmost parts are modified as

$$
\begin{aligned}
& \int_{0}^{\infty} p_{3}^{2} d p_{3}\left(g\left(p_{3}\right) \Gamma+\Delta G^{\psi \phi_{3}}\left(E q_{3}\right) g\left(p_{3}\right)\right. \\
& \left.\quad \times \Gamma\left(G_{0}^{\phi \phi \phi}\left(E q_{3} p_{3}\right) \hat{\tau}_{3}(E)+1\right)\right) G_{0}^{\phi \phi \phi}\left(E q_{3} p_{3}\right) g\left(p_{3}^{\prime}\right) .
\end{aligned}
$$

[1] S. K. Choi et al. (Belle Collaboration), Phys. Rev. Lett. 91, 262001 (2003).

[2] T. Barnes and S. Godfrey, Phys. Rev. D 69, 054008 (2004).

[3] S. K. Choi et al., Phys. Rev. D 84, 052004 (2011).

[4] C. Hanhart, Yu. S. Kalashnikova, A. E. Kudryavtsev, and A. V. Nefediev, Phys. Rev. D 85, 011501(R) (2012).

[5] M. B. Voloshin and L. B. Okun', JETP Lett. 23, 333 (1976).

[6] N. A. Tornqvist, Phys. Rev. Lett. 67, 556 (1991).

[7] E. S. Swanson, Phys. Lett. B 588, 189 (2004).

[8] N. A. Tornqvist, Phys. Lett. B 590, 209 (2004).

[9] M. B. Voloshin, Phys. Lett. B 604, 69 (2004).

[10] E. Braaten and M. Kusunoki, Phys. Rev. D 72, 054022 (2005).

[11] D. Gamermann and E. Oset, Eur. Phys. J. A 33, 119 (2007).

[12] X. Liu, Y.-R. Liu, W.-Z. Deng, and S.-L. Zhu, Phys. Rev. D 77, 034003 (2008).

[13] Y.-R. Liu, X. Liu, W.-Z. Deng, and S.-L. Zhu, Eur. Phys. J. C 56, 63 (2008).

[14] C. E. Thomas and F. E. Close, Phys. Rev. D 78, 034007 (2008).

[15] Y. Dong, A. Faessler, T. Gutsche, and V. E. Lyubovitskij, Phys. Rev. D 77, 094013 (2008).

[16] D. Gamermann, J. Nieves, E. Oset, and E. R. Arriola, Phys. Rev. D 81, 014029 (2010).
[17] F. Close and C. Downum, Phys. Rev. Lett. 102, 242003 (2009).

[18] F. Close, C. Downum, and C. E. Thomas, Phys. Rev. D 81, 074033 (2010).

[19] S. Yasui, S. Ohkoda, Y. Yamaguchi, K. Sudoh, and A. Hosaka, Few-Body Syst. 54, 1023 (2013).

[20] Y. Yamaguchi, S. Yasui, Y. Kikuchi, and A. Hosaka, JPS Conf. Proc. 1, 013073 (2014).

[21] M. Suzuki, Phys. Rev. D 72, 114013 (2005).

[22] S. Fleming, M. Kusunoki, T. Mehen, and U. van Kolck, Phys. Rev. D 76, 034006 (2007).

[23] E. Braaten, M. Lu, and J. Lee, Phys. Rev. D 76, 054010 (2007).

[24] A. A. Filin, A. Romanov, V. Baru, C. Hanhart, Yu. S. Kalashnikova, A. E. Kudryavtsev, U. G. Meissner, and A. V. Nefediev, Phys. Rev. Lett. 105, 019101 (2010).

[25] V. Baru, A. A. Filin, C. Hanhart, Yu. S. Kalashnikova, A. E. Kudryavtsev, and A. V. Nefediev, Phys. Rev. D 84, 074029 (2011)

[26] Yu. S. Kalashnikova and A. V. Nefediev, Pisma Zh. Eksp. Teor. Fiz. 97, 76 (2013) [JETP Lett. 97, 70 (2013)].

[27] E. Braaten, Phys. Rev. D 91, 114007 (2015).

[28] N. Brambilla et al., Eur. Phys. J. C 71, 1534 (2011).

[29] S. L. Olsen, PoS Bormio2015, 050 (2015). 
[30] H.-X. Chen, W. Chen, X. Liu, and S.-L. Zhu, Phys. Rep. 639, 1 (2016).

[31] A. Hosaka, T. Iijima, K. Miyabayashi, Y. Sakai, and S. Yasui, Prog. Theor. Exp. Phys. 2016, 062 C01 (2016).

[32] R. L. Jaffe, Phys. Rev. Lett. 38, 195 (1977); 38, 617(E) (1977).

[33] A. Feliciello and T. Nagae, Rep. Prog. Phys. 78, 096301 (2015).

[34] A. Gal, E. V. Hungerford, and D. J. Millener, Rev. Mod. Phys. 88, 035004 (2016).

[35] R. J. Oakes, Phys. Rev. 131, 2239 (1963).

[36] F. Dyson and N. H. Xuong, Phys. Rev. Lett. 13, 815 (1964).

[37] M. Bashkanov et al., Phys. Rev. Lett. 102, 052301 (2009).

[38] P. Adlarson et al. (WASA-at-COSY Collaboration), Phys. Rev. Lett. 106, 242302 (2011).

[39] P. Adlarson et al. (WASA-at-COSY Collaboration), Phys. Lett. B 721, 229 (2013).

[40] P. Adlarson et al. (WASA-at-COSY Collaboration), Phys. Rev. C 86, 032201(R) (2012).

[41] P. Adlarson et al. (WASA-at-COSY Collaboration), Phys. Rev. C 91, 015201 (2015).

[42] P. Adlarson et al. (WASA-at-COSY Collaboration), Phys. Rev. Lett. 112, 202301 (2014).

[43] P. Adlarson et al. (WASA-at-COSY Collaboration), Phys. Rev. C 90, 035204 (2014).
[44] A. Gal and H. Garcilazo, Phys. Rev. Lett. 111, 172301 (2013).

[45] A. Gal and H. Garcilazo, Nucl. Phys. A 928, 73 (2014).

[46] M. N. Platonova and V. I. Kukulin, Nucl. Phys. A 946, 117 (2016).

[47] R. G. Newton, Scattering Theory of Waves and Particles, reprint, 2nd ed. (Dover, Mineola, NY, 2002), pp. $x x+745$, with list of errata prepared for this edition by the author.

[48] A. Matsuyama and K. Yazaki, Nucl. Phys. A 534, 620 (1991).

[49] E. O. Alt, P. Grassberger, and W. Sandhas, Nucl. Phys. B 2, 167 (1967).

[50] W. Glockle and R. Brandenburg, Phys. Rev. C 27, 83 (1983).

[51] H. Feshbach, Ann. Phys. 5, 357 (1958).

[52] H. Feshbach, Ann. Phys. 19, 287 (1962); 281, 519 (2000).

[53] R. D. Amado, in Elementary Particle Physics and Scattering Theory: Proceedings of the 10th Brandeis University Summer Institute in Theoretical Physics, Vols. 1-2: Waltham, MA, 1967, Vol. V2 (Gordon and Breach, New York, 1970), pp. 1-123.

[54] B. C. Pearce and I. R. Afnan, Phys. Rev. C 30, 2022 (1984).

[55] I. R. Afnan, Austral. J. Phys. 44, 201 (1991).

[56] A. Konishi, O. Morimatsu, and S. Yasui, arXiv:1705.02569.

[57] C. Hanhart, J. R. Pelaez, and G. Rios, Phys. Lett. B 739, 375 (2014). 